TRANSACTIONS OF THE

AMERICAN MATHEMATICAL SOCIETY

Volume 350, Number 10, October 1998, Pages 4127-4148

S $0002-9947(98) 02326-5$

\title{
THE EXT CLASS OF AN APPROXIMATELY INNER AUTOMORPHISM
}

\author{
AKITAKA KISHIMOTO AND ALEX KUMJIAN
}

\begin{abstract}
Let $A$ be a simple unital $A \mathbf{T}$ algebra of real rank zero. It is shown below that the range of the natural map from the approximately inner automorphism group to $K K(A, A)$ coincides with the kernel of the map $K K(A, A) \rightarrow \bigoplus_{i=0}^{1} \operatorname{Hom}\left(K_{i}(A), K_{i}(A)\right)$.
\end{abstract}

\section{INTRODUCTION AND PRELIMINARIES}

1.1. An automorphism $\alpha$ of a unital $\mathrm{C}^{*}$-algebra $A$ is said to be an approximately inner automorphism if there is a sequence of unitaries $u_{n} \in A$ such that $\alpha(a)=$ $\lim \operatorname{Ad} u_{n}(a)$ for all $a \in A$. It follows that the induced map on $K_{*}(A)$ is the identity map; there is, however, an invariant of $K$-theoretical interest which can occur. Nontrivial extensions may arise in the six-term periodic sequence for the $K$-theory of the mapping torus. We show below that every extension does arise if $A$ is a simple unital $A \mathbf{T}$ algebra of real rank zero. As an immediate corollary we obtain a stronger form of Elliott's classification theorem for such algebras: an invertible $K K$ element that preserves positivity and the class of the unit lifts to an isomorphism.

1.2. Recall that a unital $\mathrm{C}^{*}$-algebra $A$ is said to be a unital $A \mathbf{T}$ algebra if it is expressible as the inductive limit of finite direct sums of algebras of the form $M_{n} \otimes C(\mathbf{T})$ with unital embeddings. Let $A$ be a unital $A \mathbf{T}$ algebra and let $\alpha$ be an approximately inner automorphism of $A$. The mapping torus of $\alpha$ is the $\mathrm{C}^{*}$-algebra

$$
M_{\alpha}=\{x \in C[0,1] \otimes A: \alpha(x(0))=x(1)\} .
$$

It will be convenient to identify $S A$, the suspension of $A$, with the kernel of the map of evaluation at zero, $e_{0}: M_{\alpha} \rightarrow A$. From the short exact sequence:

$$
0 \rightarrow S A \rightarrow M_{\alpha} \stackrel{e_{0}}{\rightarrow} A \rightarrow 0
$$

one obtains the usual six-term periodic exact sequence:

$$
\begin{array}{cccc}
K_{0}(S A) & \rightarrow K_{0}\left(M_{\alpha}\right) & \rightarrow & K_{0}(A) \\
\uparrow & & \downarrow \\
K_{1}(A) & \leftarrow K_{1}\left(M_{\alpha}\right) & \leftarrow & K_{1}(S A)
\end{array}
$$

In identifying $K_{i}(S A)$ with $K_{i+1}(A)$ the index maps become id $-\alpha_{*}$ (cf. [B, 10.4.1]); further, as $\alpha$ is an approximately inner automorphism one has $\alpha_{*}=$ id and the index

Received by the editors August 5, 1996.

1991 Mathematics Subject Classification. Primary 46L40; Secondary 46L80, 46L35.

(C)1998 American Mathematical Society 
maps are zero. Consequently, the six-term periodic exact sequence reduces to a pair of short exact sequences:

$$
0 \rightarrow K_{i+1}(A) \rightarrow K_{i}\left(M_{\alpha}\right) \rightarrow K_{i}(A) \rightarrow 0
$$

for $i=0,1$. Let $\eta_{i}(\alpha)$ denote the class of this sequence in $\operatorname{Ext}\left(K_{i}(A), K_{i+1}(A)\right)$. Since $A$ is an inductive limit of type I $\mathrm{C}^{*}$-algebras, $A$ is in the bootstrap class, and thus the universal coefficient theorem of Rosenberg and Schochet (see [RS, 1.17]) applies: that is, the following is exact:

$$
\begin{aligned}
0 & \rightarrow \bigoplus_{i=0}^{1} \operatorname{Ext}\left(K_{i}(A), K_{i+1}(A)\right) \stackrel{\delta}{\longrightarrow} K K(A, A) \\
& \stackrel{\gamma}{\longrightarrow} \bigoplus_{i=0}^{1} \operatorname{Hom}\left(K_{i}(A), K_{i}(A)\right) \rightarrow 0 .
\end{aligned}
$$

Rørdam observed (see $[\mathrm{R} \varnothing, \mathrm{p} .435])$ that $\delta\left(\eta_{0}(\alpha), \eta_{1}(\alpha)\right)=1-[\alpha]$, where $[\alpha]$ denotes the class of $\alpha$ in $K K(A, A)$. Since the product of any two elements in $\operatorname{ker} \gamma$ is 0 ([RS, 7.10]), one has $\eta_{i}(\alpha \beta)=\eta_{i}(\alpha)+\eta_{i}(\beta)$ for approximately inner automorphisms $\alpha, \beta$.

1.3. Since $A$ is a unital $A \mathbf{T}$ algebra, both $K_{0}(A)$ and $K_{1}(A)$ are torsion free and so the extensions above are pure (i.e. locally trivial). Given a pair of countable abelian groups $P, Q$; if $Q=\lim Q_{n}$ (with connecting maps $f_{n}: Q_{n} \rightarrow Q_{n+1}$ ) one has the following short exact sequence for $\operatorname{Ext}(Q, P)$ (cf. [Ro]):

$$
0 \rightarrow \lim ^{1} \operatorname{Hom}\left(Q_{n}, P\right) \rightarrow \operatorname{Ext}(Q, P) \rightarrow \lim _{\leftarrow} \operatorname{Ext}\left(Q_{n}, P\right) \rightarrow 0 .
$$

If $Q$ is torsion free, the $Q_{n}$ may be chosen to be isomorphic to $\mathbf{Z}^{k_{n}}$; in this case $\operatorname{Ext}\left(Q_{n}, P\right)=0$ and one has the isomorphism

$$
\lim _{\leftarrow}^{1} \operatorname{Hom}\left(Q_{n}, P\right) \cong \operatorname{Ext}(Q, P) .
$$

In showing that every Ext class arises from the $K K$-class of an approximately inner automorphism, it will be useful to have an explicit form for this isomorphism. Note that $\lim ^{1} \operatorname{Hom}\left(Q_{n}, P\right)$ may be identified with the cokernel of the map, $\prod_{n} \operatorname{Hom}\left(Q_{n}, \overleftarrow{P)} \rightarrow \prod_{n} \operatorname{Hom}\left(Q_{n}, P\right)\right.$, where $\left(g_{n}\right) \mapsto\left(g_{n}-g_{n+1} f_{n}\right)$. Given $\left(g_{n}\right)$, we construct an extension

$$
0 \rightarrow P \rightarrow E \rightarrow Q \rightarrow 0
$$

as follows: set $E_{n}=P \oplus Q_{n}$ and define $h_{n}: E_{n} \rightarrow E_{n+1}$ by $h_{n}(p, q)=$ $\left(p+g_{n}(q), f_{n}(q)\right)$; then $E=\lim E_{n}$ gives the desired extension. If $P=\lim P_{n}$ then, by passing to a suitable subsequence (and relabeling), one has $E \cong \lim P_{n} \oplus Q_{n}$.

We will have occasion to consider inductive systems of short exact sequences; such an inductive system will consist of a sequence of short exact sequences:

$$
0 \rightarrow P_{n} \rightarrow E_{n} \rightarrow Q_{n} \rightarrow 0
$$

together with maps between them such that the following diagram commutes:

$$
\begin{aligned}
& 0 \rightarrow P_{n} \quad \rightarrow \quad E_{n} \quad \rightarrow \quad Q_{n} \quad \rightarrow \quad 0 \\
& 0 \rightarrow \begin{array}{c}
\downarrow \\
\downarrow \\
P_{n+1}
\end{array} \rightarrow E_{n+1}^{\downarrow} \rightarrow Q_{n+1}^{\downarrow} \rightarrow 0
\end{aligned}
$$

Note that the limit of the inductive system is again a short exact sequence:

$$
0 \rightarrow P \rightarrow E \rightarrow Q \rightarrow 0
$$


1.4. In $\S 2$ we compute the class of the two extensions arising from the $K$-theory of the mapping torus for certain approximately inner automorphisms of a unital $A \mathbf{T}$ algebra (see Theorem 2.4). Here we do not assume that the $\mathrm{C}^{*}$-algebra $A$ is simple or has real rank zero. The unitality is not essential either if we define an automorphism to be approximately inner when it is so in the algebra $A^{+}$obtained by adjoining a unit to $A$, since $A^{+}$is again an $A \mathbf{T}$ algebra (see [E, $\left.\S 1\right]$ ). The maps that determine the extensions keep track of the $K_{1}$-class of $u_{n+1} u_{n}{ }^{*}$ for the various partial embeddings (this gives the maps $K_{0}\left(A_{n}\right) \rightarrow K_{1}(A)$ ) as well as the Bott number for approximately commuting unitaries (see [Ex]): it may be assumed that $u_{n+1}$ approximately commutes with the canonical central unitaries of $A_{n}$ (this gives the maps $\left.K_{1}\left(A_{n}\right) \rightarrow K_{0}(A)\right)$.

Definition. Given two unitary $n \times n$ matrices $U$ and $V$ with $\left\|V U V^{*} U^{*}-1\right\|<2$, there is a selfadjoint matrix $H$ with $\|H\|<1 / 2$ such that $V U V^{*} U^{*}=e^{2 \pi i H}$. Then define the Bott number of the pair by $B(U, V)=\operatorname{Tr}(H)$.

Since $\operatorname{det}\left(V U V^{*} U^{*}\right)=1$, it follows that $\operatorname{Tr}(H) \in \mathbf{Z}$. Note that $B(U, V)=$ $\omega(U, V)$, where $\omega(U, V)$ is the winding number of the loop (see [Ex, Lemma 3.1] and $[\mathrm{EL}])$ :

$$
t \mapsto \operatorname{det}((1-t) U V+t V U)=\operatorname{det}(U V) \operatorname{det}\left(1-t+t V U V^{*} U^{*}\right)
$$

Thus, the Bott number is invariant under homotopy of pairs of unitaries for which the norm of the commutator is less than 2. Moreover, if $\left\|V_{i} U V_{i}^{*} U^{*}-1\right\|<1$ for $i=1,2$, then $B\left(U, V_{1} V_{2}\right)=B\left(U, V_{1}\right)+B\left(U, V_{2}\right)$.

1.5. We proceed to the main result, Theorem 3.1, in $\S 3$. Let $A$ be a simple unital $A \mathbf{T}$ algebra of real rank zero; we show for $i=0,1$ that, given

$$
\eta \in \operatorname{Ext}\left(K_{i}(A), K_{i+1}(A)\right)
$$

there is an approximately inner automorphism $\alpha$ for which $\eta_{i}(\alpha)=\eta$ and $\eta_{i+1}(\alpha)=$ 0 . The proof is divided into the two cases $i=0,1$ and proceeds through a sequence of lemmas; one must for example show that an extension is expressible as an inductive limit of groups of the form $\mathbf{Z}^{k_{n}} \oplus \mathbf{Z}^{k_{n}}$ in a way that makes all relevant diagrams commute.

1.6. With $A$ as above, $K_{0}(A)$ is a simple dimension group and $K_{1}(A)$ is a (countable) torsion free group. $K_{i}(A)$ is thus expressible as the inductive limit of a system

$$
\mathbf{Z}^{k_{1}} \stackrel{\chi_{1}^{i}}{\longrightarrow} \mathbf{Z}^{k_{2}} \stackrel{\chi_{2}^{i}}{\longrightarrow} \mathbf{Z}^{k_{3}} \rightarrow \cdots
$$

with $\chi_{n}^{0}(j, k)>0$ for all $j, k$ and $n$. We may assume that image of $\mathbf{Z}^{k_{1}}$ contains the class of the unit $\left[1_{A}\right] \in K_{0}(A)$. We show below that, under mild assumptions on the $\chi_{n}^{i}, A$ is expressible as an inductive limit of certain maps $\varphi_{n}: A_{n} \rightarrow A_{n+1}$, where $K_{i}\left(A_{n}\right)=\mathbf{Z}^{k_{n}}$ and $\left(\varphi_{n}\right)_{*}=\left(\chi_{n}^{0}, \chi_{n}^{1}\right)$ and the partial embeddings of $\varphi_{n}$ are given by the standard maps described below. These maps are closely related to those arising in the path model constructed by Deaconu in [D].

Let $k_{0}, k_{1}$ be integers with $k_{0} \gg 0$. Define $\varphi_{k_{0}, k_{1}}: C(\mathbf{T}) \rightarrow M_{k_{0}} \otimes C(\mathbf{T})$ by

$$
\varphi_{k_{0}, k_{1}}(u)(z)=\left(\begin{array}{cccc}
0 & 0 & \cdots & z^{k_{1}} \\
1 & 0 & \cdots & 0 \\
& \ddots & \ddots & \\
0 & \cdots & 1 & 0
\end{array}\right)
$$


for $k_{1} \neq 0$, and define $\varphi_{k_{0}, 0}$ by

$$
\varphi_{k_{0}, 0}(u)(z)=\left(\begin{array}{cc}
\varphi_{\left\lfloor k_{0} / 2\right\rfloor, 1} & 0 \\
0 & \varphi_{\left\lceil k_{0} / 2\right\rceil,-1}
\end{array}\right) .
$$

Note that when $K_{i}(C(\mathbf{T}))$ is identified with $\mathbf{Z},\left(\varphi_{k_{0}, k_{1}}\right)_{*}$ is given by multiplication by $k_{i}$. We will refer to $\varphi_{k_{0}, k_{1}}$ as a standard embedding of type $\left(k_{0}, k_{1}\right)$. Note that under the assumption $k_{0} \gg 0$ the image of the unitary generator $v$ of $C(\mathbf{T})$ has small spectral variation in $M_{k_{0}} \otimes C(\mathbf{T})$; indeed, if $k_{1} \neq 0$ the spectrum of $\varphi_{k_{0}, k_{1}}(v)(z)$ consists of the $k_{0}^{t h}$ roots of $z^{k_{1}}$, where $z \in \mathbf{T}$. To avoid cumbersome notation we let $\varphi_{k_{0}, k_{1}}$ also denote the map $M_{n} \otimes C(\mathbf{T}) \rightarrow M_{n} \otimes M_{k_{0}} \otimes C(\mathbf{T})$ (where $\left.x \otimes a \mapsto x \otimes \varphi_{k_{0}, k_{1}}(a)\right)$.

A unitary $u \in M_{n} \otimes C(\mathbf{T})$ is said to be normal if there are integers $p, q$ with $p \neq 0, q>0$ and $q \mid n$ such that the eigenvalues of $u_{t}$ (for $t \in \mathbf{T}=\mathbf{R} / \mathbf{Z}$ ) are $e^{2 \pi i(p t+r) / q}$ for $r=0,1, \ldots, q-1$, and each eigenvalue occurs with multiplicity $n / q$. Observe that the spectral variation of $u$,

$$
\sup _{s, t \in \mathbf{T}} \operatorname{dist}\left(\sigma\left(u_{s}\right), \sigma\left(u_{t}\right)\right),
$$

is $\pi / q$, where $\sigma(u)$ denotes the spectrum of the unitary $u$ counted with multiplicity and distance is given by the arc length metric. Note that $\varphi_{q, p}(v) \in M_{q} \otimes C(\mathbf{T})$ (where $v$ is the unitary generator of $C(\mathbf{T})$ ) is normal with parameters $p, q$.

Fact. Suppose $v \in M_{n} \otimes C(\mathbf{T})$ is a normal unitary with parameters $p, q$, and

$$
\varphi_{k_{0}, k_{1}}: M_{n} \otimes C(\mathbf{T}) \rightarrow M_{n} \otimes M_{k_{0}} \otimes C(\mathbf{T})
$$

is a standard embedding with $k_{1} \neq 0$. Then $\varphi_{k_{0}, k_{1}}(v)$ is normal in $M_{n} \otimes M_{k_{0}} \otimes C(\mathbf{T})$ with parameters $p k_{1} / l, q k_{0} / l$, where $l=\operatorname{gcd}\left(p, k_{0}\right)$. Hence if $k_{0} \gg|p|$, then $\varphi_{k_{0}, k_{1}}(v)$ has small spectral variation.

We proceed to the construction of $A$ using standard embeddings. By passing to a subsequence if necessary we may assume that $M\left(\chi_{n+1}^{0}\right) \geq \max \left(2 L\left(\chi_{n}^{1}\right), 4\right)$, where

$$
M(\chi)=\min _{i, j} \chi_{i, j} \text { and } L(\chi)=\max _{i, j}\left|\chi_{i, j}\right|
$$

for a matrix $\chi$. Choose a preimage of the unit $\left[1_{A}\right] \in K_{0}(A)$ in $\mathbf{Z}^{k_{1}}$ and denote its $i^{\text {th }}$ coordinate by $[1, i]$; set $[n+1, i]=\sum_{j} \chi_{n}^{0}(i, j)[n, j], A_{n}=\bigoplus_{k=1}^{k_{n}} M_{[n, k]} \otimes C(\mathbf{T})$ and define $\varphi_{n}: A_{n} \rightarrow A_{n+1}$ to be the block diagonal sum of standard embeddings of type $\left(\chi_{n}^{0}(i, j), \chi_{n}^{1}(i, j)\right)$ (that is, the partial embedding from the $j^{\text {th }}$ summand of $A_{n}$ to the $i^{\text {th }}$ summand of $A_{n+1}$ is of the above type). Since the partial embedding from a central summand in $A_{n}$ to a central summand in $A_{m}$ with $m>n$ is a sum of composites of standard embeddings, a central unitary is mapped to a sum of normal unitaries which by the above assumption must have uniformly small spectral variation; it decreases by a factor of two at least when embedded into the next level (we require $M\left(\chi_{n+1}^{0}\right) \geq 4$ to deal with the case $\chi_{n}^{1}(i, j)=0$ ). Then $\lim A_{n}$ is a simple $A \mathbf{T}$ algebra of real rank zero (small spectral variation guarantees real rank zero - see [BBEK, 1.3]). Hence, by Elliott's classification theorem for simple real rank zero $A \mathbf{T}$ algebras (cf. [E, 7.1]), $A \cong \lim A_{n}$.

1.7. In Corollary 3.13 we establish a sharper version of Elliott's theorem: given two unital simple $A \mathbf{T}$ algebras of real rank zero, $A$ and $B$, then an invertible element in $K K(A, B)$ which preserves positivity and the class of the unit lifts to an isomorphism. 
1.8. The attempt to understand an invariant for approximately inner automorphisms of simple unital $A \mathbf{T}$ algebras of real rank zero introduced by Elliott and Rørdam (see [ER, 4.5]) stimulated our interest in the question addressed in the present work; their analysis of the invariant for the case of the Bunce-Deddens algebra (see [ER, 4.12i]) was particularly helpful. We thank George Elliott for several valuable conversations; we also wish to thank the staff of the Fields Institute, where much of this work was done, for their hospitality.

1.9. Some notational conventions: as usual we let $\mathbf{N}, \mathbf{Z}, \mathbf{R}$ and $\mathbf{T}$ denote the natural numbers, the integers, the reals, and the complex numbers of unit modulus. For $m \in \mathbf{N}$, let $\left\{e_{i}\right\}$ denote the canonical basis in $\mathbf{Z}^{m}$; a homomorphism $\chi: \mathbf{Z}^{n} \rightarrow \mathbf{Z}^{m}$ will be confused with the $m \times n$ matrix $\chi_{i, j}($ or $\chi(i, j))$ for which $\chi\left(e_{j}\right)=\sum_{i} \chi_{i, j} e_{i}$.

\section{The extension Class of an approximately inNer aUtomorphism}

2.1. Let $A$ be an $A \mathbf{T}$ algebra given as the limit of an inductive system of circle algebras $\left\{A_{n}, \varphi_{m n}\right\}$, where $A_{n}=\bigoplus_{k=1}^{k_{n}} A_{n, k}, A_{n, k}=M_{[n, k]} \otimes C(\mathbf{T})$ and $\varphi_{m n}$ : $A_{n} \rightarrow A_{m}$ is a unital homomorphism for $m>n$; to simplify notation write $\varphi_{n}$ in place of $\varphi_{n+1, n}$. Also, let $\bar{\varphi}_{n}$ denote the canonical homomorphism from $A_{n}$ to $A$. Let $v_{n, k}$ be the unitary in $A_{n}$ which restricts to the canonical central unitary in the $k^{t h}$ summand and the identity in the other summands. Let $\left\{e_{i j}^{n, k}\right\}$ denote the canonical family of matrix units for the $k^{t h}$ summand and let $p_{n, k}$ denote the projection onto the $k^{\text {th }}$ summand ( $\operatorname{so} p_{n, k}=\sum_{i} e_{i i}^{n, k}$ ). Set $R_{n}=\left\{e_{i j}^{n, k}: 1 \leq i, j \leq\right.$ $\left.[n, k], 1 \leq k \leq k_{n}\right\} \cup\left\{v_{n, k}: 1 \leq k \leq k_{n}\right\}$ and $S_{n}=R_{n} \cup \varphi_{n-1}\left(S_{n-1}\right)$, where $S_{0}$ is the empty set.

2.2. We consider a family of approximately inner automorphisms and determine the resulting Ext classes in the following theorem. Let $\alpha_{n}=\operatorname{Ad} u_{n}$ be an inner automorphism of $A_{n}$, where $u_{n}$ is a unitary in $A_{n} ; \alpha_{n}$ and $\delta_{n}>0$ are chosen inductively as follows: If $\varphi$ and $\varphi^{\prime}$ are homomorphisms from $A_{n}$ to $A_{n+1}$ such that $\left\|\varphi(x)-\varphi^{\prime}(x)\right\|<\delta_{n}$ for $x \in R_{n}$, then $\left\|\varphi(x)-\varphi^{\prime}(x)\right\|<2^{-n}$ for $x \in S_{n}$. Choose $\alpha_{n+1}$ such that

$$
\begin{aligned}
& \left\|\alpha_{n+1}^{l} \circ \varphi_{n}\left(e_{i j}^{n, k}\right)-\varphi_{n} \circ \alpha_{n}^{l}\left(e_{i j}^{n, k}\right)\right\|<\delta_{n} / 32[n, k] k_{n}, \\
& \left\|\alpha_{n+1}^{l} \circ \varphi_{n}\left(v_{n, k}\right)-\varphi_{n} \circ \alpha_{n}^{l}\left(v_{n, k}\right)\right\|<\delta_{n} / 32
\end{aligned}
$$

for $l= \pm 1$. Since $R_{n}$ generates $A_{n}$, the limit

$$
\lim _{m \rightarrow \infty} \bar{\varphi}_{m} \circ \alpha_{m} \circ \varphi_{m n}(x)=\lim _{m \rightarrow \infty} \operatorname{Ad} \bar{\varphi}_{m}\left(u_{m}\right)\left(\bar{\varphi}_{n}(x)\right)
$$

exists for all $x \in R_{n}$. One defines a homomorphism $\alpha: \bigcup_{n=1}^{\infty} \bar{\varphi}_{n}\left(A_{n}\right) \rightarrow A$ by

$$
\alpha\left(\bar{\varphi}_{n}(x)\right)=\lim _{m \rightarrow \infty} \bar{\varphi}_{m} \circ \alpha_{m} \circ \varphi_{m n}(x)
$$

for $x \in A_{n}$; note that $\alpha$ extends to a unital endomorphism. Since $\beta \circ \bar{\varphi}_{n}(x)=$ $\lim \bar{\varphi}_{m} \circ \alpha_{m}{ }^{-1} \circ \bar{\varphi}_{m n}(x)$ for $x \in A_{n}$ defines a unital endomorphism $\beta$ of $A$ and $\alpha \circ \beta \circ \bar{\varphi}_{n}(x)=\bar{\varphi}_{n}(x)$ for $x \in S_{n}$, it follows that $\alpha$ is an automorphism of $A$. 
2.3. We view elements of $A_{n}$ as matrix valued functions on $k_{n}$ copies of $\mathbf{T}$ (the size of the matrix may vary). Since

$$
\left\|\alpha_{n+1} \circ \varphi_{n}\left(v_{n, j}\right)-\varphi_{n}\left(v_{n, j}\right)\right\|<2^{-n},
$$

the Bott number $B\left(\varphi_{n}\left(v_{n, j}\right)\left(\iota_{i}\right), u_{n+1}\left(\iota_{i}\right)\right)$ (see 1.4) is well-defined (where $\iota_{i}$ is the basepoint of the $i^{t h}$ copy of $\mathbf{T}$ in the spectrum of $\left.A_{n+1}\right)$. For the purposes of computing the Bott number we may suppose that $u_{n+1}\left(\iota_{i}\right)$ commutes with $\varphi_{n}\left(p_{n, j}\right)\left(\iota_{i}\right)$ (by perturbing it slightly) and so regard both $\varphi_{n}\left(v_{n, j}\right)\left(\iota_{i}\right)$ and $u_{n+1}\left(\iota_{i}\right)$ as unitaries in $\varphi_{n}\left(p_{n, j}\right)\left(\iota_{i}\right) M_{[n+1, i]} \varphi_{n}\left(p_{n, j}\right)\left(\iota_{i}\right)$. Since the image of $A_{n, j}$ in

$$
\varphi_{n}\left(p_{n, j}\right)\left(\iota_{i}\right) M_{[n+1, i]} \varphi_{n}\left(p_{n, j}\right)\left(\iota_{i}\right)
$$

almost commutes with $\varphi_{n}\left(v_{n, j}\right)\left(\iota_{i}\right)$ and $u_{n+1} \varphi_{n}\left(u_{n}^{*}\right)\left(\iota_{i}\right)$, it follows that

$$
B\left(\varphi_{n}\left(v_{n, j}\right)\left(\iota_{i}\right), u_{n+1}\left(\iota_{i}\right)\right)=B\left(\varphi_{n}\left(v_{n, j}\right)\left(\iota_{i}\right), u_{n+1}\left(\iota_{i}\right) \varphi_{n}\left(u_{n}^{*}\right)\left(\iota_{i}\right)\right)
$$

is a multiple of $[n, j]$. For $i=1, \ldots, k_{n+1}$ and $j=1, \ldots, k_{n}$ set

$$
\psi_{n}^{0}(i, j)=\left\{\begin{array}{cl}
B\left(\varphi_{n}\left(v_{n, j}\right)\left(\iota_{i}\right), u_{n+1}\left(\iota_{i}\right)\right) /[n, j] & \text { if } \varphi_{n}\left(p_{n, j}\right) p_{n+1, i} \neq 0, \\
0 & \text { otherwise }
\end{array}\right.
$$

(note that this is an integer) and

$$
\psi_{n}^{1}(i, j)=\left\{\begin{array}{cl}
{\left[\varphi_{n}\left(p_{n, j}\right) u_{n+1} p_{n+1, i} \varphi_{n}\left(u_{n}^{*} p_{n, j}\right)\right]_{1} /[n, j]} & \text { if } \varphi_{n}\left(p_{n, j}\right) p_{n+1, i} \neq 0, \\
0 & \text { otherwise }
\end{array}\right.
$$

where $[\cdot]_{1}$ denotes the class of an invertible element in

$$
K_{1}\left(\varphi_{n}\left(p_{n, j}\right) A_{n+1, i} \varphi_{n}\left(p_{n, j}\right)\right) \cong \mathbf{Z}
$$

Since $u_{n+1} p_{n+1, i} \varphi_{n}\left(u_{n}^{*} p_{n, j}\right)$ almost commutes with the image of $A_{n, j}=M_{[n, j]}$, $\psi_{n}^{1}(i, j)$ is again an integer (for $n$ sufficiently large).

$K_{i}(A)$ is given as the inductive limit of the sequence

$$
\mathbf{Z}^{k_{1}} \stackrel{\chi_{1}^{i}}{\longrightarrow} \mathbf{Z}^{k_{2}} \stackrel{\chi_{2}^{i}}{\longrightarrow} \mathbf{Z}^{k_{3}} \rightarrow \cdots
$$

where $\chi_{n}^{i}=\left(\varphi_{n}\right)_{*}$, viewed as a $k_{n+1} \times k_{n}$ matrix with integer entries (note that $\left.\chi_{n}^{0}(i, j) \geq 0\right)$.

2.4. Theorem. Let $A, A_{n}, \alpha, \alpha_{n}, \psi_{n}^{i}, \chi_{n}^{i}$ be as above. Then for $i=0,1$ the extension

$$
0 \quad \rightarrow \quad K_{i+1}(A) \quad \rightarrow \quad K_{i}\left(M_{\alpha}\right) \rightarrow K_{i}(A) \quad \rightarrow 0
$$

is obtained as the inductive limit of the sequence

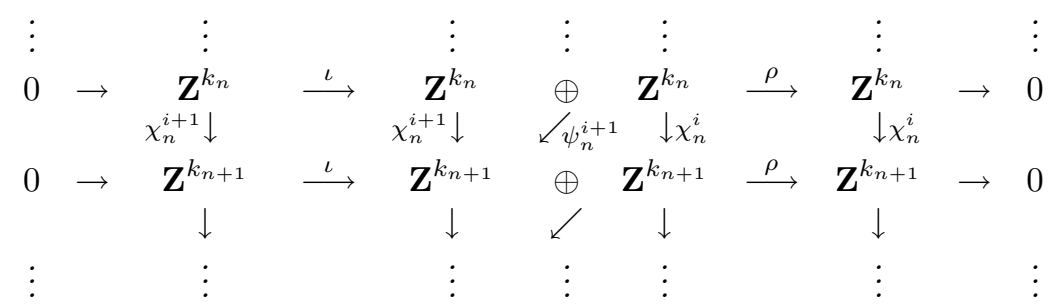

where $\iota$ is the embedding onto the first summand $\mathbf{Z}^{k_{n}}$ and $\rho$ is the projection onto the second summand $\mathbf{Z}^{k_{n}}$ for each $n$; note that in each case $K_{0}$ is regarded as an ordered abelian group (while $K_{1}$ is regarded as an abelian group). 
2.5. We proceed now to the proof of the theorem. For the inner automorphism $\alpha_{n}$ of $A_{n}$ the six-term periodic sequence for the mapping torus $M_{\alpha_{n}}$ reduces to two trivial short exact sequences:

$$
0 \rightarrow K_{i+1}\left(A_{n}\right) \rightarrow K_{i}\left(M_{\alpha_{n}}\right) \rightarrow K_{i}\left(A_{n}\right) \rightarrow 0
$$

for $i=0,1$; note that $K_{i}\left(A_{n}\right)$ is naturally isomorphic to $\mathbf{Z}^{k_{n}}$ for $i=0,1$. The identification of $K_{0}\left(M_{\alpha_{n}}\right)$ (resp. $K_{1}\left(M_{\alpha_{n}}\right)$ ) with $\mathbf{Z}^{k_{n}} \oplus \mathbf{Z}^{k_{n}}$ as ordered abelian groups (resp. abelian groups) proceeds as follows: Let $v:[0,1] \rightarrow U\left(M_{2} \otimes A_{n}\right)$ be a continuous path of unitaries with

$$
v_{0}=\left(\begin{array}{cc}
u_{n} & 0 \\
0 & u_{n}^{*}
\end{array}\right), \quad v_{1}=\left(\begin{array}{cc}
1 & 0 \\
0 & 1
\end{array}\right) .
$$

For $x \oplus y \in M_{\alpha_{n}} \oplus M_{\alpha_{n}^{-1}}$ set

$$
(x \oplus y)^{\sim}(t)=v_{t}\left(\begin{array}{cc}
x(t) & 0 \\
0 & y(t)
\end{array}\right) v_{t}^{*} .
$$

One checks that

$$
(x \oplus y)^{\sim}(0)=(x \oplus y)^{\sim}(1) ;
$$

thus $(x \oplus y)^{\sim}$ may be regarded as an element of $C(\mathbf{T}) \otimes M_{2} \otimes A_{n}$. This defines an embedding of $M_{\alpha_{n}} \oplus M_{\alpha_{n}^{-1}}$ into $C(\mathbf{T}) \otimes M_{2} \otimes A_{n}$. Since the image of the map $M_{\alpha_{n}} \rightarrow C(\mathbf{T}) \otimes M_{2} \otimes A_{n}$ (by $x \mapsto(x \oplus 0)^{\sim}$ ) is a full hereditary subalgebra, we obtain an isomorphism,

$$
K_{*}\left(M_{\alpha_{n}}\right) \simeq K_{*}\left(C(\mathbf{T}) \otimes M_{2} \otimes A_{n}\right) .
$$

To identify $K_{i}\left(C(\mathbf{T}) \otimes M_{2} \otimes A_{n}\right)$ with $\mathbf{Z}^{k_{n}} \oplus \mathbf{Z}^{k_{n}}$ it suffices to identify $K_{i}(C(\mathbf{T}) \otimes$ $\left.M_{2} \otimes A_{n, k}\right)$ with $\mathbf{Z} \oplus \mathbf{Z}$ in the usual way - note that

$$
C(\mathbf{T}) \otimes M_{2} \otimes A_{n, k}=C(\mathbf{T}) \otimes M_{2} \otimes M_{[n, k]} \otimes C(\mathbf{T}) \simeq M_{2[n, k]} \otimes C\left(\mathbf{T}^{2}\right) .
$$

The class of a unitary $u \in M_{2[n, k]} \otimes C\left(\mathbf{T}^{2}\right)$ is identified with $\left([u(\cdot, 1)]_{1},[u(1, \cdot)]_{1}\right)$ (where the first variable is identified with the parameter in the mapping torus construction) and the class of a projection, $p \in M_{2[n, k]} \otimes C\left(\mathbf{T}^{2}\right)$, is identified with $(\operatorname{ch}(p), \operatorname{dim}(p))$, where $\operatorname{ch}(p)$ is the first Chern class of $p$ and $\operatorname{dim}(p)$ is the dimension of $p$. Under this identification a nonzero element $(m, n)$ is positive if and only if $n>0$.

2.6. Lemma. With identifications as above the extension

$$
0 \rightarrow K_{i+1}\left(A_{n}\right) \rightarrow K_{i}\left(M_{\alpha_{n}}\right) \rightarrow K_{i}\left(A_{n}\right) \rightarrow 0,
$$

for $i=0,1$ is isomorphic to

$$
0 \rightarrow \mathbf{Z}^{k_{n}} \stackrel{\iota}{\longrightarrow} \mathbf{Z}^{k_{n}} \oplus \mathbf{Z}^{k_{n}} \stackrel{\rho}{\longrightarrow} \mathbf{Z}^{k_{n}} \rightarrow 0
$$

where $\iota(a)=(a, 0)$ and $\rho(a, b)=b$ for $a, b \in \mathbf{Z}^{k_{n}}$.

Proof. Let $u$ be a unitary in $A_{n}$ and let $w:[0,1] \rightarrow U\left(M_{2} \otimes A_{n}\right)$ be a continuous path of unitaries with

$$
w_{0}=\left(\begin{array}{cc}
u & 0 \\
0 & u^{*}
\end{array}\right), \quad w_{1}=\left(\begin{array}{cc}
1 & 0 \\
0 & 1
\end{array}\right) .
$$

Set $e_{t}=w_{t}(1 \oplus 0) w_{t}^{*}$; then $e$ is a projection in $M_{2} \otimes(S A) \sim$ and the map $K_{1}\left(A_{n}\right) \rightarrow$ $K_{0}\left(M_{\alpha_{n}}\right)$, is given by $[u] \mapsto[e]-[1]$ (see [B, 8.2.2]). By the above identification the second component of $[e]-[1]$ zero. Since the second component of $K_{0}\left(M_{\alpha_{n}}\right)$ 
corresponds to dimension, the map $K_{0}\left(M_{\alpha_{n}}\right) \rightarrow K_{0}\left(A_{n}\right)$ is just the projection onto the second component.

Let $p$ be a projection in $A_{n}$. Set $u_{t}=\left(e^{2 \pi i t}-1\right) p+1$. Then $u$ is a unitary in $(S A) \sim M_{\alpha_{n}}$, and $[p]$ maps to $[u]$ under the map $K_{0}\left(A_{n}\right) \rightarrow K_{1}\left(M_{\alpha_{n}}\right)$. Set $\tilde{u}(t)=v_{t}\left(u_{t} \oplus 1\right) v_{t}^{*}$, where $\left\{v_{t}\right\}$ is as above (see equation (2)); we now compute $[\tilde{u}]_{1}$ (in $K_{1}\left(C(\mathbf{T}) \otimes M_{2} \otimes A_{n}\right)$ ). Since $\tilde{u}(1)=1$, the second component of $[\tilde{u}]$ must be zero. The winding number of

$$
t \mapsto \operatorname{det} \tilde{u}(t)\left(\iota_{i}\right)=\operatorname{det}\left(\left(e^{2 \pi i t}-1\right) p\left(\iota_{i}\right)+1\right)
$$

with $\tilde{u}(t)$ evaluated at the point $\iota_{i}$ in the spectrum of $A_{n}$ is just $\operatorname{dim} p\left(\iota_{i}\right)=$ $\operatorname{dim}\left(p \cdot p_{n, i}\right)$. Thus the first component of $[\tilde{u}]$ is equal to $[p]$. Let $u$ be a unitary in $M_{\alpha_{n}}$, and set

$$
\tilde{u}(t)=v_{t}\left(u_{t} \oplus 1\right) v_{t}^{*} .
$$

Then, since $\tilde{u}(1)=u_{1} \oplus 1,[u]=\left(*,\left[u_{1}\right]\right)$ maps to $\left[u_{1}\right]$ under the map $K_{1}\left(M_{\alpha_{n}}\right) \rightarrow$ $K_{1}\left(A_{n}\right)$.

2.7. We now define a homomorphism from $M_{\alpha_{n}}$ to $M_{\alpha_{n+1}}$. This is done by using the fact that $\varphi_{n} \circ \alpha_{n}$ and $\alpha_{n+1} \circ \varphi_{n}$ are homotopic (as homomorphisms from $A_{n}$ to $\left.A_{n+1}\right)$. The induced map, $K_{i}\left(M_{\alpha_{n}}\right) \rightarrow K_{i}\left(M_{\alpha_{n+1}}\right)$ (for $\left.i=0,1\right)$, is computed in the following lemma.

Recall that we have chosen $\left\{\alpha_{n}\right\}$ so that

$$
\begin{aligned}
& \left\|\alpha_{n+1} \circ \varphi_{n}\left(e_{i j}^{n, k}\right)-\varphi_{n} \circ \alpha_{n}\left(e_{i j}^{n, k}\right)\right\|<\delta_{n} / 32[n, k] k_{n}, \\
& \left\|\alpha_{n+1} \circ \varphi_{n}\left(v_{n, k}\right)-\varphi_{n} \circ \alpha_{n}\left(v_{n, k}\right)\right\|<\delta_{n} / 32 .
\end{aligned}
$$

We assume that $\delta_{n}>0$ is sufficiently small in the following. Set

$$
x=\sum_{i, k} \alpha_{n+1} \circ \varphi_{n}\left(e_{i i}^{n, k}\right) \varphi_{n} \circ \alpha_{n}\left(e_{i i}^{n, k}\right)
$$

then ||$x-1||<\delta_{n} / 32$, and the unitary $v_{1}=x|x|^{-1}$ in $A_{n+1}$ satisfies

$$
\operatorname{Ad} v_{1} \circ \varphi_{n} \circ \alpha_{n}\left(e_{i i}^{n, k}\right)=\alpha_{n+1} \circ \varphi_{n}\left(e_{i i}^{n, k}\right)
$$

and $\left\|v_{1}-1\right\|<\delta_{n} / 16$ (for large $n$ ). It follows that for $i \neq j$

$$
\left\|\operatorname{Ad} v_{1} \circ \varphi_{n} \circ \alpha_{n}\left(e_{i j}^{n, k}\right)-\alpha_{n+1} \circ \varphi_{n}\left(e_{i j}^{n, k}\right)\right\|<5 \delta_{n} / 32[n, k] k_{n}<5 \delta_{n} / 32 .
$$

Now set

$$
v_{2}=\sum_{i, k} \operatorname{Ad} v_{1} \circ \varphi_{n} \circ \alpha_{n}\left(e_{i 1}^{n, k}\right) \alpha_{n+1} \circ \varphi_{n}\left(e_{1 i}^{n, k}\right) ;
$$

then $v_{2}$ is a unitary such that $\left\|v_{2}-1\right\| \leq 5 \delta_{n} / 32$ and $\operatorname{Ad} v_{2} v_{1} \circ \varphi_{n} \circ \alpha_{n}\left(e_{i j}^{n, k}\right)=$ $\alpha_{n+1} \circ \varphi_{n}\left(e_{i j}^{n, k}\right)$. Since $\left\|v_{2} v_{1}-1\right\|<7 \delta_{n} / 32$, there is a self-adjoint $h \in A_{n+1}$ such that $2 \pi\|h\| \leq \delta_{n} / 4$ and $v_{2} v_{1}=e^{2 \pi i h}$. Since

$$
\left\|\operatorname{Ad} e^{2 \pi i h} \circ \varphi_{n} \circ \alpha_{n}\left(v_{n, k}\right)-\alpha_{n+1} \circ \varphi_{n}\left(v_{n, k}\right)\right\|<15 \delta_{n} / 32,
$$

there is a self-adjoint $h_{k} \in A_{n+1}$ such that $2 \pi|| h_{k} \|<16 \delta_{n} / 32$ and $e^{2 \pi i h_{k}}=\alpha_{n+1} \circ$ $\varphi_{n}\left(v_{n, k}\right) \operatorname{Ad} e^{2 \pi i h} \circ \varphi_{n} \circ \alpha_{n}\left(v_{n, k}^{*}\right)$. For $t \in[0,1]$ we define a homomorphism $\nu_{t}$ : $A_{n} \rightarrow A_{n+1}$ as follows: for $0 \leq t \leq 1 / 2$, set

$$
\nu_{t}=\operatorname{Ad} e^{4 \pi i t h} \circ \varphi_{n} \circ \alpha_{n}=\operatorname{Ad}\left(e^{4 \pi i t h} \varphi_{n}\left(u_{n}\right)\right) \circ \varphi_{n},
$$


and for $1 / 2<t \leq 1$, set

$$
\begin{aligned}
& \nu_{t}\left(e_{i j}^{n, k}\right)=\alpha_{n+1} \circ \varphi_{n}\left(e_{i j}^{n, k}\right), \\
& \nu_{t}\left(v_{n, k}\right)=e^{2 \pi i(2 t-1) h_{k}} \operatorname{Ad} e^{2 \pi i h} \circ \varphi_{n} \circ \alpha_{n}\left(v_{n, k}\right) .
\end{aligned}
$$

Note that $\nu_{t}$ is well defined, $t \mapsto \nu_{t}(x)$ is continuous for all $x \in A_{n}, \nu_{0}=\varphi_{n} \circ \alpha_{n}$ and $\nu_{1}=\alpha_{n+1} \circ \varphi_{n}$. Moreover, $\left\|\nu_{t}(x)-\varphi_{n} \circ \alpha_{n}(x)\right\| \leq 2^{-n}$ for all $x \in S_{n}$ (since $\left\|\nu_{t}(x)-\varphi_{n} \circ \alpha_{n}(x)\right\|<\delta_{n}$ for $\left.x \in R_{n}\right)$. Define $\Phi_{n}: M_{\alpha_{n}} \rightarrow M_{\alpha_{n+1}}$ by

$$
\Phi_{n}(x)(t)= \begin{cases}\varphi_{n}\left(x\left(\frac{t}{1-2^{-n}}\right)\right), & 0 \leq t \leq 1-2^{-n}, \\ \nu_{2^{n}\left(t-1+2^{-n}\right)}(x(0)), & 1-2^{-n}<t \leq 1 .\end{cases}
$$

2.8. Lemma. Let $\Phi_{n}$ be as above. Then, for $i=0,1,\left(\Phi_{n}\right)_{*}: K_{i}\left(M_{\alpha_{n}}\right) \rightarrow$ $K_{i}\left(M_{\alpha_{n+1}}\right)$ is given by

$$
\begin{array}{ccr}
\mathbf{Z}^{k_{n}} & \oplus & \mathbf{Z}^{k_{n}} \\
\chi_{n}^{i+1} \downarrow & \swarrow_{n}^{i+1} & \downarrow \chi_{n}^{i} \\
\mathbf{Z}^{k_{n+1}} & \oplus & \mathbf{Z}^{k_{n+1}}
\end{array}
$$

Proof. We deal with the case $i=0$ first. Let $u \in A_{n}$ be a unitary and $\left\{w_{t}\right\}$ a continuous path of unitaries in $M_{2} \otimes A_{n}$ from $u \oplus u^{*}$ to 1 . Set $e_{t}=w_{t}(1 \oplus 0) w_{t}^{*}$. Since $e_{0}=1 \oplus 0, \Phi_{n}(e)$ is equivalent to the projection defined by $t \mapsto \varphi_{n}\left(e_{t}\right)$, which is defined by $\varphi_{n}(u)$ in the same way as $e$ is defined by $u$. Since $\Phi_{n}(1)=1$ and $[u]$ is mapped to $[e]-[1]$, this shows that $\chi_{n}^{1}$ gives the map from the first summand of $K_{0}\left(M_{\alpha_{n}}\right)$ to the first summand of $K_{0}\left(M_{\alpha_{n+1}}\right)$ (a similar argument shows that $\chi_{n}^{0}$ is the analogous map for $i=1$ ).

Let $p_{n, k}$ be the central projection in $A_{n}$ as before. Regarding $p_{n, k}$ as a projection in $M_{\alpha_{n}}$, we have

$$
\left[p_{n, k}\right]=\left(\left[u_{n} p_{n, k}\right],[n, k]\right) \in \mathbf{Z} \oplus \mathbf{Z} \subset \mathbf{Z}^{k_{n}} \oplus \mathbf{Z}^{k_{n}},
$$

where $\mathbf{Z} \oplus \mathbf{Z}$ is mapped to the $k^{t h}$ summand of $(\mathbf{Z} \oplus \mathbf{Z})^{k_{n}}=\mathbf{Z}^{k_{n}} \oplus \mathbf{Z}^{k_{n}}$. Let $\left\{v_{t}\right\}$ be a continuous path of unitaries in $M_{2} \otimes A_{n+1}$ from $u_{n+1} \oplus u_{n+1}^{*}$ to 1 . Since $u_{n+1} \oplus u_{n+1}^{*}$ almost commutes with $\varphi_{n}\left(p_{n, k}\right) \oplus \varphi_{n}\left(p_{n, k}\right)$, we can assume that $v_{t}$ almost commutes with $\varphi_{n}\left(p_{n, k}\right) \oplus \varphi_{n}\left(p_{n, k}\right)$ for all $t \in[0,1]$. It follows that the class of $\Phi_{n}\left(p_{n, k}\right)$ in $\mathbf{Z}^{k_{n+1}} \oplus \mathbf{Z}^{k_{n+1}}$ is the equivalence class of $t \mapsto v_{t}\left(\varphi_{n}\left(p_{n, k}\right) \oplus 0\right) v_{t}^{*}$ composed with a short path from $\varphi_{n}\left(p_{n, k}\right) \oplus 0$ to $\alpha_{n+1} \circ \varphi_{n}\left(p_{n, k}\right) \oplus 0$. Since this corresponds to the invertible element $\varphi_{n}\left(p_{n, k}\right) u_{n+1} \varphi_{n}\left(p_{n, k}\right)+1-\varphi_{n}\left(p_{n, k}\right)$, the first component of $\left[\Phi_{n}\left(p_{n, k}\right)\right]$ is

$$
\begin{aligned}
{\left[\varphi_{n}\left(p_{n, k}\right) u_{n+1} \varphi_{n}\left(p_{n, k}\right)\right]_{1} } & =\left[\varphi_{n}\left(p_{n, k}\right) u_{n+1} \varphi_{n}\left(u_{n}^{*}\right) \varphi_{n}\left(p_{n, k}\right)\right]_{1}+\left[\varphi_{n}\left(u_{n}\right) \varphi_{n}\left(p_{n, k}\right)\right]_{1} \\
& =\left(\psi_{n}^{1}(i, k)[n, k]+\chi_{n}^{1}(i, k)\left[u_{n} p_{n, k}\right]\right)_{i},
\end{aligned}
$$

where $[\cdot]_{1}$ denotes the $K_{1}$-class of an invertible element. The second component of $\left[\Phi_{n}\left(p_{n, k}\right)\right]$ is the $K_{0}$-class of $\varphi_{n}\left(p_{n, k}\right)$, i.e. $\left(\chi_{n}^{0}(i, k)[n, k]\right)$. This takes care of the case $i=0$.

Regarding $v_{n, k}$ as an element of $M_{\alpha_{n}}$, it follows that $\left[\Phi_{n}\left(v_{n, k}\right)\right]$ is the class of

$$
U: t \mapsto v_{t}\left(\Phi_{n}\left(v_{n, k}\right)(t) \oplus 1\right) v_{t}^{*}
$$

in $K_{1}\left(C(\mathbf{T}) \otimes M_{2} \otimes A_{n+1}\right)$. Evaluate $U$ at $\iota_{i}$ (the base point of the $i^{t h}$ copy of $\mathbf{T}$ in the spectrum of $A_{n+1}$ ) and let $w_{i}$ be the winding number of

$$
\begin{aligned}
t & \mapsto \operatorname{det}\left(v_{t}\left(\iota_{i}\right)\left(\Phi_{n}\left(v_{n, k}\right)\left(t, \iota_{i}\right) \oplus 1\right) v_{t}\left(\iota_{i}\right)^{*}\right) \\
& =\operatorname{det}\left(\Phi_{n}\left(v_{n, k}\right)\left(t, \iota_{i}\right) \oplus 1\right) .
\end{aligned}
$$


By the definition of $\Phi_{n}\left(v_{n, k}\right), w_{i}$ is equal to $\operatorname{Tr}\left(h_{k}\left(\iota_{i}\right)\right)$, where $h_{k}$ is defined by

$$
e^{2 \pi i h_{k}}=\alpha_{n+1} \circ \varphi_{n}\left(v_{n, k}\right) \operatorname{Ad} e^{2 \pi i h} \circ \varphi_{n} \circ \alpha_{n}\left(v_{n, k}^{*}\right)
$$

with $h_{k}$ small. Since

$$
e^{-2 \pi i h} e^{2 \pi i h_{k}} e^{2 \pi i h}=e^{-2 \pi i h} u_{n+1} \cdot \varphi_{n}\left(v_{n, k}\right) \cdot\left(e^{-2 \pi i h} u_{n+1}\right)^{*} \cdot \varphi_{n}\left(v_{n, k}\right)^{*},
$$

if follows that $w_{i}$ is equal to

$$
B\left(\varphi_{n}\left(v_{n, k}\right)\left(\iota_{i}\right), e^{-2 \pi i h\left(\iota_{i}\right)} u_{n+1}\left(\iota_{i}\right)\right)=B\left(\varphi_{n}\left(v_{n, k}\right)\left(\iota_{i}\right), u_{n+1}\left(\iota_{i}\right)\right)=\psi_{n}^{0}(i, k)[n, k] .
$$

Note that $[U(0)]=\left[\varphi_{n}\left(v_{n, k}\right)\right]$; thus, $\left[\Phi_{n}\left(v_{n, k}\right)\right]=\left(\psi_{n}^{0}(i, k)[n, k], \chi_{n}^{1}(i, k)[n, k]\right)_{i}$. Since $\left[v_{n, k}\right]=(0,[n, k]) \in \mathbf{Z} \oplus \mathbf{Z} \subset \mathbf{Z}^{k_{n}} \oplus \mathbf{Z}^{k_{n}}$, the proof in the case $i=1$ is complete.

2.9. Lemma. For any $x \in M_{\alpha_{n}}$, the limit

$$
\lim _{m \rightarrow \infty} \bar{\varphi}_{n+m} \circ \Phi_{n+m-1} \circ \cdots \circ \Phi_{n}(x)
$$

exists in $C[0,1] \otimes A$ and defines a homomorphism of $M_{\alpha_{n}}$ to $M_{\alpha}$.

Proof. Given $x \in M_{\alpha_{n}}$, then for all $t \in[0,1], \bar{\varphi}_{n+m} \circ \Phi_{n+m-1} \circ \cdots \circ \Phi_{n}(x)(t)$ converges, say to $\tilde{x}(t)$; one has $\tilde{x}(1)=\alpha(\tilde{x}(0))$ and $\tilde{x}(0)=x(0)$. It also follows that convergence is uniform on $[0, t]$ for all $t<1$. For $x \in S_{n}$ the family of functions, $\left\{\bar{\varphi}_{n+m} \circ \Phi_{n+m-1} \circ \cdots \circ \Phi_{n}(x)(t): m>0\right\}$ is uniformly continuous in a neighbourhood of $t=1$. Hence convergence is uniform for all $x \in M_{\alpha_{n}}$.

2.10. We conclude the proof of the theorem. By the previous lemma we have a homomorphism $\mu_{n}: M_{\alpha_{n}} \rightarrow M_{\alpha}$ such that $\mu_{n}=\mu_{n+1} \circ \Phi_{n}$. Let $E$ denote the $\mathrm{C}^{*}$-inductive $\operatorname{limit} \lim _{n} M_{\alpha_{n}}$; there is a homomorphism $\mu: E \rightarrow M_{\alpha}$ such that $\mu_{n}=\mu \circ \bar{\Phi}_{n}$, where $\bar{\Phi}_{n}: M_{\alpha_{n}} \rightarrow E$ is the canonical map. We show that $\mu$ is an isomorphism.

If $\operatorname{ker} \mu \neq 0$, then $\operatorname{ker} \mu \cap \operatorname{im} \bar{\Phi}_{n} \neq 0$ for some $n$. Let $x \in M_{\alpha_{n}}$ be such that $\mu \circ \bar{\Phi}_{n}(x)=\mu_{n}(x)=0$; then, since $\mu_{n}(x)(t)=\bar{\varphi}_{n}\left(x\left(t / s_{n}\right)\right)$ for $0 \leq t \leq s_{n}$, where $s_{n}=\prod_{n}^{\infty}\left(1-2^{-k}\right)>1-2^{-n+1}$, if follows that $\bar{\varphi}_{n}(x(t))=0$, and thus $\bar{\Phi}_{n}(x)(t)=0$ for $0 \leq t \leq 1-2^{-n+1}$. Since $\bar{\Phi}_{n}(x)=\bar{\Phi}_{m} \circ \Phi_{m n}(x)$ for $m>n$, it follows that $\bar{\Phi}_{n}(x)=0$; thus, $\mu$ is injective.

Let $x \in M_{\alpha}$ with $x(0) \in \bar{\varphi}_{k}\left(S_{k}\right)$ for some $k$ and $\epsilon>0$. There exist an $n$ and $y \in C[0,1] \otimes A_{n}$ such that $\left\|x(t)-\bar{\varphi}_{n}(y(t))\right\|<\epsilon$. Since

$$
\begin{aligned}
&\left\|\bar{\varphi}_{m} \circ \alpha_{m} \circ \varphi_{m n}(y(0))-\bar{\varphi}_{n}(y(1))\right\| \\
& \leq\left\|\bar{\varphi}_{m} \circ \alpha_{m} \circ \varphi_{m n}(y(0))-\alpha \circ \bar{\varphi}_{m} \circ \varphi_{m n}(y(0))\right\| \\
& \quad+\left\|\alpha \circ \bar{\varphi}_{n}(y(0))-\alpha(x(0))\right\|+\left\|x(1)-\bar{\varphi}_{n}(y(1))\right\| \\
& \leq \epsilon_{m}+2 \epsilon
\end{aligned}
$$

where $\epsilon_{m} \rightarrow 0$ as $m \rightarrow \infty$, we may take a small modification of $y$ around $t=1$ and obtain, for sufficiently large $m>n, y \in C[0,1] \otimes A_{m}$ such $y(0) \in S_{n}, \bar{\varphi}_{m}(y(0))=$ $x(0), y \in M_{\alpha_{m}}$, and $\left\|x(t)-\bar{\varphi}_{m}(y(t))\right\|<3 \epsilon$. If $m$ is sufficiently large we have that $\left\|\bar{\varphi}_{m}(y(t))-\mu_{m}(y)(t)\right\|<7 \epsilon$, since $\mu_{m}(y)$ is obtained by extending $t \mapsto \bar{\varphi}_{m}(y(t))$ beyond $t=1$ up to $1 / s_{m}$ with $s_{m}$ defined above and rescaling it; the extended part is within a sphere of radius $2^{-m+1}$ centered at $\bar{\varphi}_{m}(y(1))$. Thus we have that $\left\|x-\mu_{m}(y)\right\|<11 \epsilon$. This implies that $\mu$ is surjective. 


\section{ReAlization OF AN EXTENSION ClASS BY AN APPROXIMATELY INNER AUTOMORPHISM}

3.1. The proof of the following theorem will be divided into two cases; the case $i=1$ will be dealt with in sections 3.8 and 3.9 and the case $i=0$ will be handled in 3.11 .

Theorem. Let $A$ be a simple unital AT algebra of real rank zero, and let $i=0$ or 1. Then, given an extension

$$
0 \rightarrow K_{i+1}(A) \rightarrow E \rightarrow K_{i}(A) \quad \rightarrow \quad 0
$$

there is an approximately inner automorphism $\alpha$ of $A$ such that

$$
0 \rightarrow K_{i+1}(A) \rightarrow K_{i}\left(M_{\alpha}\right) \rightarrow K_{i}(A) \rightarrow 0
$$

is equivalent to the above extension and

$$
0 \quad \rightarrow \quad K_{i}(A) \quad \rightarrow \quad K_{i+1}\left(M_{\alpha}\right) \rightarrow K_{i+1}(A) \rightarrow 0
$$

is a trivial extension. That is, for every element $\eta \in \operatorname{Ext}\left(K_{i}(A), K_{i+1}(A)\right)$ there is an approximately inner automorphism $\alpha$ of $A$ such that $\eta_{i}(\alpha)=\eta$ and $\eta_{i+1}(\alpha)=0$.

3.2. Lemma. Given $\chi \in M_{m n}(\mathbf{Z})$ and a subgroup $W$ of $\mathbf{Z}^{m}$ with $\operatorname{im} \chi \cap W=\{0\}$, there is a constant $c \geq 0$ such that for any $\psi \in M_{m n}(\mathbf{Z})$ with $\operatorname{ker} \psi \supset$ ker $\chi$ there is a $\gamma \in M_{n}(\mathbf{Z})$ such that

$$
\operatorname{ker} \gamma \supset W \quad \text { and } \quad|(\psi-\gamma \chi)(i, j)| \leq c
$$

for $i=1, \ldots, m, j=1, \ldots, n$.

Proof. Let $r=\operatorname{rank} \chi$ and let $b_{1}, \ldots, b_{n}$ be generators for $\mathbf{Z}^{n}$ such that $\chi b_{1}, \ldots, \chi b_{r}$ generate $\chi \mathbf{Z}^{n}$ and $\chi b_{i}=0$ for $i=r+1, \ldots, n$. We denote by $U$ the invertible matrix in $M_{n}(\mathbf{Z})$ defined by $\left(b_{1} \cdots b_{n}\right)$.

Let $\tilde{W}$ be a maximal subgroup of $\mathbf{Z}^{m}$ such that

$$
\tilde{W} \cap \operatorname{im} \chi=\{0\}, \tilde{W} \supset W .
$$

Then $\tilde{W}$ is isomorphic to $\mathbf{Z}^{m-r}$. Let $a_{i}=\chi b_{i}$ for $i=1, \ldots, r$, and let $a_{r+1}, \ldots, a_{m}$ be generators for $\tilde{W}$. Define a matrix $V \in M_{m}(\mathbf{Z})$ by $V=\left(a_{1} \cdots a_{m}\right)$. Note that the determinant $\operatorname{det} V$ is non-zero and that $|\operatorname{det} V|>1$ if $\operatorname{im} \chi+\tilde{W} \neq \mathbf{Z}^{m}$. Then $\chi$ can be expressed by

$$
\chi=V \tilde{E}_{r} U^{-1}
$$

where $\tilde{E}_{r}$ is the canonical rank $r$ matrix in $M_{m n}(\mathbf{Z})$ with 1 on the first $r$ diagonal elements and 0 elsewhere. Since $\operatorname{ker} \psi \supset \operatorname{ker} \chi, \psi$ can be expressed as

$$
\begin{aligned}
\psi & =\left(\psi\left(b_{1}\right) \cdots \psi\left(b_{r}\right) 0 \cdots 0\right) \tilde{E}_{r} U^{-1} \\
& =\left(\psi\left(b_{1}\right) \cdots \psi\left(b_{r}\right) 0 \cdots 0\right) V^{-1} \chi
\end{aligned}
$$

Note that for any $\eta_{1}, \ldots, \eta_{r}$ in $\mathbf{Z}^{m}$ the matrix

$$
\gamma=\left(\eta_{1} \cdots \eta_{r} 0 \cdots 0\right)(\operatorname{det} V) V^{-1}
$$

is such that $\gamma \in M_{m}(\mathbf{Z})$ and $\operatorname{ker} \gamma \supset \tilde{W} \supset W$. We choose $\eta_{i} \in \mathbf{Z}^{m}$ so that

$$
\left\|(\operatorname{det} V)^{-1} \psi\left(b_{i}\right)-\eta_{i}\right\|_{\infty} \leq 1 / 2 \text {, }
$$

where $\|x\|_{\infty}=\max \left|x_{j}\right|$ for $x \in \mathbf{R}^{m}$. Then defining $\gamma$ as above, we obtain that

$$
\psi-\gamma \chi=\left\{(\operatorname{det} V)^{-1}\left(\psi\left(b_{1}\right) \cdots \psi\left(b_{r}\right) 0 \cdots\right)-\left(\eta_{1} \cdots \eta_{r} 0 \cdots 0\right)\right\}(\operatorname{det} V) V^{-1} \chi
$$


and

where

$$
|(\psi-\gamma \chi)(i, j)| \leq c,
$$

$$
c=\max _{j} 1 / 2 \sum_{k, l}|\operatorname{det} V| \cdot\left|V^{-1}(k, l) \chi(l, j)\right|,
$$

which does not depend on $\psi$.

3.3. Let $G_{1}$ be a countable torsion-free abelian group. Then there exist a sequence $l_{n}$ of positive integers and an inductive system

$$
\mathbf{Z}^{l_{1}} \stackrel{\chi_{1}^{1}}{\longrightarrow} \mathbf{Z}^{l_{2}} \stackrel{\chi_{2}^{1}}{\longrightarrow} \mathbf{Z}^{l_{3}} \rightarrow \cdots
$$

for which the inductive limit is isomorphic to $G_{1}$, such that

$$
\operatorname{rank} \chi_{n+1}^{1} \chi_{n}^{1}=\operatorname{rank} \chi_{n}^{1}
$$

for $n=1,2,3, \ldots$ Since $\operatorname{rank} \chi_{n+1, m}^{1}=\operatorname{rank} \chi_{n m}^{1}$ for $n>m$, we have that ker $\chi_{n}^{1} \cap$ $\operatorname{im} \chi_{n m}^{1}=\{0\}$ for $n>m$. Thus we obtain a constant $c_{n m}=c$ for the pair $\chi_{n m}^{1}$ and ker $\chi_{n}^{1}$ by the previous lemma. We set

$$
c_{n}=\max \left\{c_{k m}: n \geq k>m\right\},
$$

which forms an increasing sequence. We always assume that $c_{n} \geq 1$.

Later, for a sequence $k_{n}$ of positive integers with $k_{n} \geq l_{n}$, we define another inductive system

$$
\mathbf{Z}^{k_{1}} \stackrel{\tilde{\chi}_{1}^{1}}{\longrightarrow} \mathbf{Z}^{k_{2}} \stackrel{\tilde{\chi}_{2}^{1}}{\longrightarrow} \mathbf{Z}^{k_{3}} \rightarrow \cdots,
$$

by extending $\chi_{n}^{1}$ with 0 's, i.e., defining $\tilde{\chi}_{n}^{1}$ by

$$
\mathbf{Z}^{k_{n}} \stackrel{\rho}{\longrightarrow} \mathbf{Z}^{l_{n}} \stackrel{\chi_{n}^{1}}{\longrightarrow} \mathbf{Z}^{l_{n+1}} \stackrel{\iota}{\longrightarrow} \mathbf{Z}^{k_{n+1}},
$$

where $\rho$ (resp. $\iota$ ) is an obvious projection (resp. embedding). The inductive limit is the same as before. Since ker $\tilde{\chi}_{n+1}^{1}=\iota\left(\operatorname{ker} \chi_{n+1}^{1}\right) \oplus \mathbf{Z}^{k_{n+1}-l_{n+1}}$ and $\operatorname{im} \tilde{\chi}_{n}^{1} \subset \mathbf{Z}^{l_{n}} \oplus 0$, we retain the same constant $c_{n}$ as before for this new inductive system.

Let $G_{0}$ be a simple dimension group other than $\mathbf{Z}$. Then there exist an increasing sequence $m_{n}$ of positive integers and an inductive system

$$
\mathbf{Z}^{m_{1}} \stackrel{\chi_{1}^{0}}{\longrightarrow} \mathbf{Z}^{m_{2}} \stackrel{\chi_{2}^{0}}{\longrightarrow} \mathbf{Z}^{m_{3}} \rightarrow \cdots,
$$

for which the inductive limit is isomorphic to $G_{0}$ (as ordered abelian groups), such that

$$
m_{n} \geq l_{n}, M\left(\chi_{n}^{0}\right) \geq n c_{n}
$$

where $M(\chi)=\min _{i, j} \chi(i, j)$ for a matrix $\chi$ with non-negative entries. The extra conditions placed on the inductive system can be handled easily.

3.4. Lemma. Given an extension

$$
0 \rightarrow K_{0}(A) \stackrel{\iota}{\longrightarrow} E \stackrel{q}{\longrightarrow} K_{1}(A) \rightarrow 0
$$

as in the theorem, there exist an increasing sequence $k_{n}$ of positive integers and inductive systems

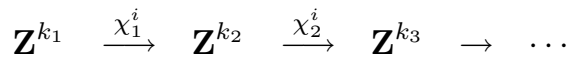

for $i=0,1$ and

$$
\mathbf{Z}^{k_{1}} \oplus \mathbf{Z}^{k_{1}} \stackrel{\psi_{1}}{\longrightarrow} \mathbf{Z}^{k_{2}} \oplus \mathbf{Z}^{k_{2}} \stackrel{\psi_{2}}{\longrightarrow} \mathbf{Z}^{k_{3}} \oplus \mathbf{Z}^{k_{3}} \rightarrow \cdots
$$


such that $M\left(\chi_{n}^{0}\right) \geq n c_{n}$ with $c_{n}$ defined for the sequence $\chi_{n}^{1}$ as in 3.3, $\psi_{n}$ is of the form $\psi_{n}(a, b)=\left(\chi_{n}^{0}(a)+\psi_{n}^{0}(b), \chi_{n}^{1}(b)\right), \operatorname{ker} \psi_{n}^{0} \supset \operatorname{ker} \chi_{n}^{1}, \operatorname{rank} \chi_{n+1}^{1} \chi_{n}^{1}=\operatorname{rank} \chi_{n}^{1}$, and the given short exact sequence is isomorphic to the inductive limit of the sequence

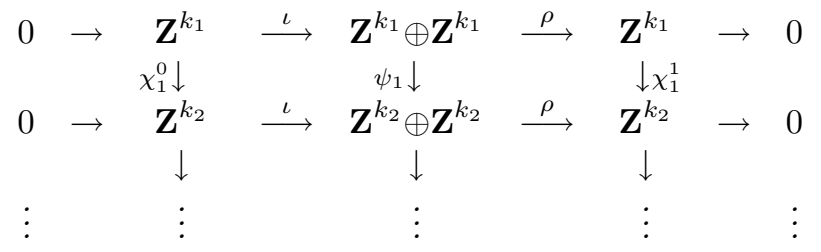

We may also assume that $M\left(\chi_{n+1}^{0}\right) \geq \max \left(2 L\left(\chi_{n}^{1}\right), 4\right)$ for all $n$.

Proof. For $G_{i}=K_{i}(A)$ we choose inductive systems as in $3.3, \mathbf{Z}^{m_{1}} \rightarrow \mathbf{Z}^{m_{2}} \rightarrow \cdots$ for $i=0$ and $\mathbf{Z}^{l_{1}} \rightarrow \mathbf{Z}^{l_{2}} \rightarrow \cdots$ for $i=1$, with all the properties specified there. We shall choose a subsequence $k(n)$ of positive integers and homomorphisms $\zeta_{n}$ : $\mathbf{Z}^{m_{k(n)}} \oplus \mathbf{Z}^{l_{n}} \rightarrow E, \quad \psi_{n}: \mathbf{Z}^{m_{k(n)}} \oplus \mathbf{Z}^{l_{n}} \rightarrow \mathbf{Z}^{m_{k(n+1)}} \oplus \mathbf{Z}^{l_{n+1}}$ for each $n$ such that the diagrams

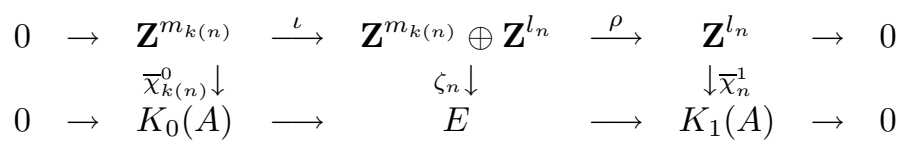

and

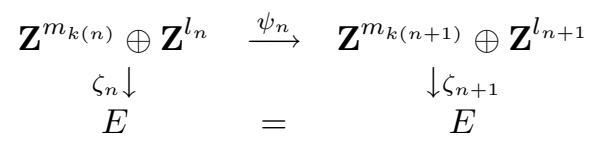

are commutative, $M\left(\chi_{k(n+1) k(n)}^{0}\right) \geq n c_{n}\left(\right.$ and $M\left(\chi_{k(n+1) k(n)}^{0}\right) \geq \max \left(2 L\left(\chi_{n-1}^{1}\right), 4\right)$, for $n>1)$ and

$$
\operatorname{ker} \psi_{n}^{0} \supset \operatorname{ker} \chi_{n}^{1}, \quad \operatorname{ker} \zeta_{n} \supset \operatorname{ker}\left(\bar{\chi}_{k(n)}^{0} \oplus \bar{\chi}_{n}^{1}\right),
$$

where $\psi_{n}(a, b)=\left(\chi_{k(n+1) k(n)}^{0}(a)+\psi_{n}^{0}(b), \chi_{n}^{1}(b)\right)$. Once this is done, we set $k_{n}=$ $m_{k(n)}\left(\geq l_{n}\right)$ and replace the inductive system $\mathbf{Z}^{l_{1}} \rightarrow \mathbf{Z}^{l_{2}} \rightarrow \cdots$ for $K_{1}(A)$ by

$$
\mathbf{Z}^{k_{1}} \stackrel{\chi_{1}^{1}}{\longrightarrow} \mathbf{Z}^{k_{2}} \rightarrow \cdots
$$

as specified in 3.3. We also redefine $\zeta_{n}, \psi_{n}$ in the obvious way. The required properties are retained under this change, and thus the proof will be complete.

We prove the above assertion by induction. Let $k(1)=1$. Since im $\bar{\chi}_{1}^{1}$ is torsionfree, it is isomorphic to $\mathbf{Z}^{r}$ for some $r \leq l_{1}$. Hence we can find a homomorphism $\phi$ of im $\bar{\chi}_{1}^{1}$ into $E$ such that $q \circ \phi=$ id and define $\zeta_{1}$ by

$$
\zeta_{1}(a, b)=\iota \circ \bar{\chi}_{1}^{0}(a)+\phi \circ \bar{\chi}_{1}^{1}(b) .
$$

Then the required properties for $\zeta_{1}$ are immediate. Suppose that we have constructed $k(1), \ldots, k(n), \zeta_{1}, \ldots, \zeta_{n}$, and $\psi_{1}, \ldots, \psi_{n-1}$ with the required properties. We then find a homomorphism $\phi: \operatorname{im} \bar{\chi}_{n+1}^{1} \rightarrow E$ such that $q \circ \phi=$ id. Choose a basis $b_{1}, \ldots, b_{l_{n}} \in \mathbf{Z}^{l_{n}}$ so that $\bar{\chi}_{n}^{1}\left(b_{1}\right), \ldots, \bar{\chi}_{n}^{1}\left(b_{r}\right)$ generates $\operatorname{im} \bar{\chi}_{n}^{1} \cong \mathbf{Z}^{r}$ and $\bar{\chi}_{n}^{1}\left(a_{i}\right)=0$ for $r<i \leq l_{n}$. Then it follows that

$$
\zeta_{n}\left(0, b_{i}\right)-\phi \circ \bar{\chi}_{n}^{1}\left(b_{i}\right) \in \iota\left(K_{0}(A)\right) .
$$

for all $1 \leq i \leq l_{n}$ (note that $\zeta_{n}\left(0, b_{i}\right)=0=\phi \circ \bar{\chi}_{n}^{1}\left(b_{i}\right)$ for $\left.i>r\right)$. Since $\bigcup_{n=1}^{\infty} \operatorname{im} \bar{\chi}_{n}^{0}=$ $K_{0}(A)$, there is a $k(n+1)>k(n)$ such that the left hand side is contained in 
$\iota\left(\operatorname{im} \bar{\chi}_{k(n+1)}^{0}\right)$ for all $i$ - we also require that $M\left(\chi_{k(n+1) k(n)}^{0}\right) \geq \max \left(2 L\left(\chi_{n-1}^{1}\right), 4\right)$ for $n>1$.

We define $\zeta_{n+1}: \mathbf{Z}^{m_{k(n+1)}} \oplus \mathbf{Z}^{l_{n+1}} \rightarrow E$ by

$$
\zeta_{n+1}(a, b)=\iota \circ \bar{\chi}_{k(n+1)}^{0}(a)+\phi \circ \bar{\chi}_{n+1}^{1}(b) ;
$$

it follows by construction that $\operatorname{ker} \zeta_{n+1} \supset \operatorname{ker}\left(\bar{\chi}_{k(n+1)}^{0} \oplus \bar{\chi}_{n+1}^{1}\right)$. Then there exist $p_{i} \in \mathbf{Z}^{m_{k(n+1)}}$ such that

$$
\zeta_{n}\left(0, b_{i}\right)-\zeta_{n+1}\left(0, \chi_{n}^{1}\left(b_{i}\right)\right)=\zeta_{n}\left(0, b_{i}\right)-\phi \circ \bar{\chi}_{n}^{1}\left(b_{i}\right)=\iota \circ \bar{\chi}_{k(n+1)}^{0}\left(p_{i}\right) .
$$

We assume that $p_{i}=0$ for $i>r$ and define $\psi_{n+1}^{0}: \mathbf{Z}^{l_{n}} \rightarrow \mathbf{Z}^{m_{k(n+1)}}$ by

$$
\psi_{n+1}^{0}\left(b_{i}\right)=p_{i} .
$$

Since the $b_{i}$ 's form a basis, $\psi_{n+1}^{0}$ is well-defined; moreover, $\operatorname{ker} \psi_{n+1}^{0} \supset \operatorname{ker} \bar{\chi}_{n+1}^{1}=$ $\operatorname{ker} \chi_{n+1}^{1}$, where the last equality follows from the assumption $\operatorname{rank} \chi_{n+1}^{1} \chi_{n}^{1}=$ rank $\chi_{n}^{1}$. Since $k(n+1) \geq n+1$ and hence $c_{k(n+1)} \geq c_{n+1}$ (from the definition of $c_{n}$ ), it follows that

$$
M\left(\chi_{k(n+1)}^{0}\right) \geq k(n+1) c_{k(n+1)} \geq(n+1) c_{n+1},
$$

where the first inequality follows from the choice made at the beginning of the proof. Since the commutativity of the diagrams follows immediately, this completes the induction.

3.5. By passing to a subsequence one may further assume that $M\left(\chi_{n}^{0}\right)>L_{n} c_{n}$ for arbitrarily large $L_{n}$. First note that for $n>m$,

$$
\psi_{n m}(a, b)=\psi_{n-1} \circ \cdots \circ \psi_{m}(a, b)=\left(\chi_{n m}^{0}(a)+\psi_{n m}^{0}(b), \chi_{n m}^{1}(b)\right),
$$

where $\psi_{n m}^{0}: \mathbf{Z}^{k_{m}} \rightarrow \mathbf{Z}^{k_{n+1}}$ is defined by

$$
\psi_{n m}^{0}(b)=\sum_{k=m}^{n-1} \chi_{n, k+1}^{0} \circ \psi_{k} \circ \chi_{k m}^{1}(b) .
$$

Thus it follows that $\operatorname{ker} \psi_{n m}^{0} \supset \operatorname{ker} \bar{\chi}_{m}^{1}=\operatorname{ker} \chi_{m}^{1}$. In this way we show that by passing to a subsequence $n_{j}$ in the situation of 3.4 all the algebraic conditions are retained and the estimate on $M\left(\chi_{n}^{0}\right)$ is improved as follows:

$$
M\left(\chi_{n_{j+1} n_{j}}^{0}\right) \geq M\left(\chi_{n_{j+1}-1}^{0}\right) \geq\left(n_{j+1}-1\right) c_{n_{j+1}-1},
$$

where $\left(c_{n_{j+1}-1}\right)$ can be regarded as $\left(c_{j}\right)$ for the sequence $\left(\chi_{n_{j+1} n_{j}}^{1}\right)$ and $n_{j+1}-1$ can be made arbitrarily large.

3.6. Lemma. Suppose that the extension

$$
0 \rightarrow K_{0}(A) \stackrel{\iota}{\longrightarrow} E \stackrel{q}{\longrightarrow} K_{1}(A) \rightarrow 0
$$

is obtained as the inductive limit of

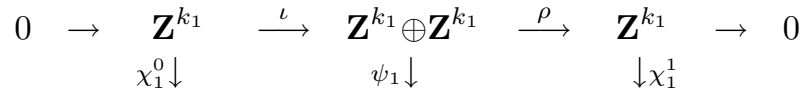

$$
\begin{aligned}
& 0 \rightarrow \mathbf{Z}^{k_{2}} \stackrel{\iota}{\longrightarrow} \mathbf{Z}^{k_{2}} \oplus \mathbf{Z}^{k_{2}} \stackrel{\rho}{\longrightarrow} \mathbf{Z}^{k_{2}} \rightarrow 0
\end{aligned}
$$


where $\psi_{n}$ is of the form $\psi_{n}(a, b)=\left(\chi_{n}^{0}(a)+\psi_{n}^{0}(b), \chi_{n}^{1}(b)\right)$. Let $\gamma_{n} \in M_{k_{n}}(\mathbf{Z})$ be given for all $n \geq 1$ and define a homomorphism $\varphi_{n}: \mathbf{Z}^{k_{n}} \oplus \mathbf{Z}^{k_{n}} \rightarrow \mathbf{Z}^{k_{n+1}} \oplus \mathbf{Z}^{k_{n+1}}$ in the same way as $\psi_{n}$ with $\psi_{n}^{0}$ replaced by

$$
\varphi_{n}^{0}=\psi_{n}^{0}+\chi_{n}^{0} \gamma_{n}-\gamma_{n+1} \chi_{n}^{1} .
$$

Then the extension obtained as the inductive limit of the above system with $\varphi_{n}$ in place of $\psi_{n}$ is isomorphic to the original extension.

Proof. Define a homomorphism $\nu_{n}: \mathbf{Z}^{k_{n}} \oplus \mathbf{Z}^{k_{n}} \rightarrow \mathbf{Z}^{k_{n}} \oplus \mathbf{Z}^{k_{n}}$ by

$$
\nu_{n}(a, b)=\left(a+\gamma_{n}(b), b\right) \text {. }
$$

Then $\nu_{n+1} \circ \varphi_{n}=\psi_{n} \circ \nu_{n}$ by computation. Thus $\left(\nu_{n}\right)$ induces an isomorphism of the inductive limit $E^{\prime}$ for $\left(\varphi_{n}\right)$ onto $E$ as required. One can show this by direct computation, or it also follows from the following commutative diagram by knowing that both rows are exact:

$$
\begin{aligned}
& 0 \rightarrow K_{0}(A) \rightarrow E^{\prime} \rightarrow K_{1}(A) \rightarrow 0 \\
& 0 \rightarrow K_{0}(A) \rightarrow E \rightarrow K_{1}(A) \rightarrow 0
\end{aligned}
$$

3.7. Lemma. Let $\varphi$ be the standard $n$-times around embedding of $C(\mathbf{T})$ into $M_{n} \otimes$ $C(\mathbf{T})$ and let $u$ be the canonical unitary of $C(\mathbf{T})$. Then for any $k \in\{0,1, \cdots, n-1\}$ there is a unitary $v \in M_{n} \otimes C(\mathbf{T})$ with $[v]=0$ such that $v \varphi(u) v^{*}=e^{2 \pi i k / n} \varphi(u)$.

Proof. Recall that $\varphi$ has the form

$$
\varphi(u)(z)=\left(\begin{array}{cccc}
0 & 0 & \cdots & z \\
1 & 0 & \cdots & 0 \\
& \ddots & \ddots & \\
0 & \cdots & 1 & 0
\end{array}\right),
$$

and define

$$
v=\left(\begin{array}{cccc}
\omega^{0} & 0 & \ldots & 0 \\
0 & \omega^{1} & \cdots & 0 \\
& & \ddots & \\
0 & 0 & \cdots & \omega^{n-1}
\end{array}\right),
$$

where $\omega=e^{2 \pi i / n}$. Then $\operatorname{Ad} v(\varphi(u))=\omega \varphi(u)$.

3.8. Proof of the theorem for the case $i=1$. By Lemma 3.4 we may express the short exact sequence.

$$
0 \rightarrow K_{0}(A) \stackrel{\iota}{\longrightarrow} E \stackrel{q}{\longrightarrow} K_{1}(A) \quad \rightarrow \quad 0
$$

as the limit of an inductive system of short exact sequences:

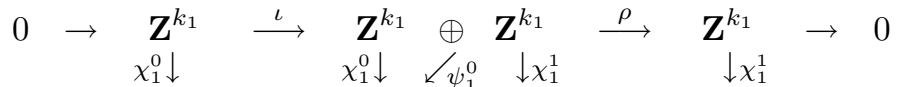

$$
\begin{aligned}
& 0 \rightarrow \mathbf{Z}^{k_{2}} \stackrel{\iota}{\longrightarrow} \mathbf{Z}^{k_{2}} \oplus \mathbf{Z}^{k_{2}} \stackrel{\rho}{\longrightarrow} \mathbf{Z}^{k_{2}} \rightarrow 0
\end{aligned}
$$


where $M\left(\chi_{n}^{0}\right)>L_{n} c_{n}$ for all $n$ and some constant $L_{n} \geq n$, $\operatorname{ker} \psi_{n}^{0} \supset \operatorname{ker} \chi_{n}^{1}$, $\operatorname{rank} \chi_{n+1}^{1} \chi_{n}^{1}=\operatorname{rank} \chi_{n}^{1}$ and $M\left(\chi_{n+1}^{0}\right) \geq \max \left(2 L\left(\chi_{n}^{1}\right), 4\right)$ for all $n$. Note by 3.5 that these properties hold with arbitrarily large $L_{n}$ when passing to a subsequence.

We choose a subsequence of the above inductive system, replace $\psi_{n}^{0}$ so that $\left|\psi_{n}^{0}(i, j)\right| \leq c_{n}$ holds (Lemma 3.2) without changing the inductive limit (Lemma 3.6), and construct circle algebras $A_{n}=\bigoplus_{k=1}^{k_{n}} M_{[n, k]} \otimes C(\mathbf{T})$ for each level of the subsequence, embeddings $\varphi_{n}: A_{n} \rightarrow A_{n+1}$ by using standard embeddings (see 1.6), and unitaries $u_{n} \in A_{n}$ such that $\varphi_{n}$ induces $\chi_{n}^{i}: K_{i}\left(A_{n}\right) \rightarrow K_{i}\left(A_{n+1}\right)$, $\operatorname{Ad} u_{n+1} \circ \varphi_{n} \approx \varphi_{n} \circ \operatorname{Ad} u_{n}$ as described in the first theorem (which depends on how small $\left|\psi_{n}^{0}(i, j)\right| / M\left(\chi_{n}^{0}\right) \leq L_{n}^{-1}$ is $),\left[u_{n+1}, \varphi_{n}\left(p_{n, j}\right)\right]=0$,

$$
\left[u_{n+1} p_{n+1, i} \varphi_{n}\left(u_{n}^{*} p_{n, j}\right)\right]_{1}=0
$$

in $K_{1}\left(\varphi_{n}\left(p_{n, j}\right) A_{n+1, i} \varphi_{n}\left(p_{n, j}\right)\right)$ and

$$
B\left(\varphi_{n}\left(v_{n, j}\right)\left(\iota_{i}\right), u_{n+1}\left(\iota_{i}\right)\right)=\psi_{n}^{0}(i, j)[n, j] .
$$

Let $A_{\infty}$ denote the inductive limit of $A_{1} \rightarrow A_{2} \rightarrow A_{3} \rightarrow \ldots$; then, by Elliott's classification theorem (cf. [E, 7.1]), $A_{\infty}$ is isomorphic to $A$ (by 1.6 the condition $M\left(\chi_{n+1}^{0}\right) \geq \max \left(2 L\left(\chi_{n}^{1}\right), 4\right)$ for all $n$ guarantees that $A_{\infty}$ has real rank zero), and the limit $\alpha=\lim \operatorname{Ad} \bar{\varphi}_{n}\left(u_{n}\right)$ defines the desired automorphism $\alpha$ of $A_{\infty}$. Note that $\eta_{0}(\alpha)=0$ by the above properties of $\left[u_{n}\right]$.

Let $u_{1}$ be an arbitrary unitary in $A_{1}$ with $\left[u_{1}\right]=0$. The construction now proceeds inductively; suppose that $A_{1}, \ldots, A_{n}, \varphi_{1}, \ldots, \varphi_{n-1}, \delta_{1}, \ldots, \delta_{n-1}$, and $u_{1}, \ldots, u_{n}$ have been chosen so that condition (1) on $\alpha_{m+1} \circ \varphi_{m} \approx \varphi_{m} \circ \alpha_{m}$ holds with $\alpha_{m}=\operatorname{Ad} u_{m}$. Recall that $S_{m+1}=R_{m+1} \cup \varphi_{m}\left(S_{m}\right)$ and $R_{m}$ are the generators for $A_{m}$.

3.9. We note the following lemma, without giving its proof.

Lemma. Let $F$ be a finite subset of $A_{n}$. For any $\epsilon>0$ there is a $\delta>0$ such that for any two homomorphisms $\varphi, \varphi^{\prime}$ from $A_{n}$ into another $C^{*}$-algebra $B$, if $\left\|\varphi(x)-\varphi^{\prime}(x)\right\|<\delta$ for all $x \in R_{n}$, then $\left\|\varphi(x)-\varphi^{\prime}(x)\right\|<\epsilon$ for $x \in F$.

By applying the above lemma for $F=S_{n}$ and $\epsilon=2^{-n}$ we obtain $\delta=\delta_{n}>0$. Then, by passing to a subsequence (see 3.5 ) and modifying $\psi_{n}^{0}, \psi_{n+1}^{0}$ by using 3.2 and 3.6, we may assume that

$$
2 \pi\left(L\left(\psi_{n}^{0}\right)+1\right) / M\left(\chi_{n}^{0}\right)<\delta_{n} / 32,
$$

where $L(\psi)=\max _{i, j}|\psi(i, j)|$. Note that the modified $\psi_{n+1}^{0}$ satisfies the condition $\operatorname{ker} \psi_{n+1}^{0} \supset \operatorname{ker} \chi_{n+1}^{1}$. Define an embedding $\varphi_{n}: A_{n} \rightarrow A_{n+1}$ so that the partial embedding from $A_{n, j}$ to $A_{n+1, i}$ is a standard embedding of type $\left(\chi_{n}^{0}(i, j), \chi_{n}^{1}(i, j)\right)$ (see 1.6). Using 3.7 choose a unitary

$$
v_{n+1} \in \varphi_{n}\left(\bigoplus_{j=1}^{k_{n}} M_{[1, j]} \otimes 1\right)^{\prime} \cap \bigoplus_{i=1}^{k_{n+1}} M_{[n+1, i]} \otimes 1 \subset A_{n+1}
$$

such that

$$
\operatorname{Ad} v_{n+1} \circ \varphi_{n}\left(v_{n j}\right) p_{n+1, i}=e^{2 \pi i \psi_{n}^{0}(i, j) / \chi_{n}^{0}(i, j)} \varphi_{n}\left(v_{n j}\right) p_{n+1, i}
$$

in the case $\chi_{n}^{1}(i, j) \neq 0$, and

$$
\operatorname{Ad} v_{n+1} \circ \varphi_{n}\left(v_{n j}\right) p_{n+1, i} \cong e^{2 \pi i a_{1} / b_{1}} \varphi_{b_{1}, 1}\left(v_{n j}\right) \oplus e^{2 \pi i a_{2} / b_{2}} \varphi_{b_{2},-1}\left(v_{n j}\right)
$$


in the case $\chi_{n}^{1}(i, j)=0$, where $\varphi_{n}\left(v_{n j}\right) \cong \varphi_{b_{1}, 1}\left(v_{n j}\right) \oplus \varphi_{b_{2},-1}\left(v_{n j}\right), a_{1}=\left\lfloor\psi_{n}^{0}(i, j) / 2\right\rfloor$, $b_{1}=\left\lfloor\chi_{n}^{0}(i, j) / 2\right\rfloor, a_{1}+a_{2}=\psi_{n}^{0}(i, j)$, and $b_{1}+b_{2}=\chi_{n}^{0}(i, j)$ (see 1.6). In either case it follows that $\left[v_{n+1} \varphi_{n}\left(p_{n, j}\right)\right]_{1}=0$ in $K_{1}\left(\varphi_{n}\left(p_{n, j}\right) A_{n+1} \varphi_{n}\left(p_{n, j}\right)\right)$, and

$$
\begin{aligned}
& B\left(\varphi_{n}\left(v_{n j}\right)\left(\iota_{i}\right), u_{n+1}\left(\iota_{i}\right)\right)=\psi_{n}^{0}(i, j)[n, j], \\
& \left\|\operatorname{Ad} v_{n+1} \circ \varphi_{n}\left(v_{n, j}\right)-\varphi_{n}\left(v_{n, j}\right)\right\|<\delta_{n} / 32, \\
& \operatorname{Ad} v_{n+1} \circ \varphi_{n}\left(e_{i j}^{n, k}\right)=\varphi_{n}\left(e_{i j}^{n, k}\right) .
\end{aligned}
$$

Setting $u_{n+1}=v_{n+1} \varphi_{n}\left(u_{n}\right)$ completes the induction.

3.10. Lemma. Given an extension as in the theorem,

$$
0 \rightarrow K_{1}(A) \stackrel{\iota}{\longrightarrow} E \stackrel{q}{\longrightarrow} K_{0}(A) \rightarrow 0,
$$

there exists an increasing sequence $k_{n}$ of positive integers and inductive systems

$$
\mathbf{Z}^{k_{1}} \stackrel{\chi_{1}^{i}}{\longrightarrow} \mathbf{Z}^{k_{2}} \stackrel{\chi_{2}^{i}}{\longrightarrow} \mathbf{Z}^{k_{3}} \rightarrow \cdots
$$

for $i=0,1$ and

$$
\mathbf{Z}^{k_{1}} \oplus \mathbf{Z}^{k_{1}} \quad \stackrel{\psi_{1}}{\longrightarrow} \mathbf{Z}^{k_{2}} \oplus \mathbf{Z}^{k_{2}} \stackrel{\psi_{2}}{\longrightarrow} \quad \mathbf{Z}^{k_{3}} \oplus \mathbf{Z}^{k_{3}} \quad \rightarrow \quad \cdots
$$

where $\chi_{n}^{0}$ is positive, and $\psi_{n}$ is of the form $\psi_{n}(a, b)=\left(\chi_{n}^{1}(a)+\psi_{n}^{1}(b), \chi_{n}^{0}(b)\right)$ for each $n$, such that the given short exact sequence is isomorphic to the inductive limit of the sequence

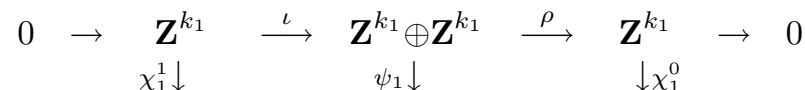

$$
\begin{aligned}
& 0 \rightarrow \mathbf{Z}^{k_{2}} \stackrel{\iota}{\longrightarrow} \mathbf{Z}^{k_{2}} \oplus \mathbf{Z}^{k_{2}} \stackrel{\rho}{\longrightarrow} \mathbf{Z}^{k_{2}} \quad \rightarrow \quad 0
\end{aligned}
$$

Before going into the proof, note that $E$ has a natural order, $a \in E$ is positive if $q(a)$ is a non-zero positive element of $K_{0}(A)$ or $a=0$, and that $E$ is a dimension group with respect to this order. Define an order on $\mathbf{Z}^{k_{n}} \oplus \mathbf{Z}^{k_{n}}$ by $(a, b) \geq 0$ if $b \geq 0, b \neq 0$ or $(a, b)=0$; observe that $\psi_{n}$ preserves order and that the inductive limit is isomorphic to $E$ as an ordered abelian group. This is how $K_{0}\left(M_{\alpha}\right)$ is obtained as an inductive limit in 2.4 .

Proof. There is an increasing sequence $\left\{l_{n}\right\}$ and inductive systems

$$
\mathbf{Z}^{l_{1}} \stackrel{\xi_{1}^{i}}{\longrightarrow} \mathbf{Z}^{l_{2}} \stackrel{\xi_{2}^{i}}{\longrightarrow} \mathbf{Z}^{l_{3}} \rightarrow \cdots
$$

with limit $K_{i}(A)$ for $i=0,1$ (as an ordered group for $i=0$ ).

First, we find a homomorphism $\zeta_{1}$ such that the diagram

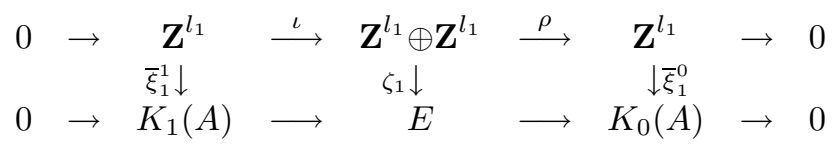


is commutative. Set $k_{1}=l_{1}, m(1)=2$ and $\theta_{1}^{i}=\bar{\xi}_{1}^{i}$; suppose that we have an increasing sequence $\left\{k_{1}, \ldots, k_{n}\right\}$ of positive integers and commutative diagrams:

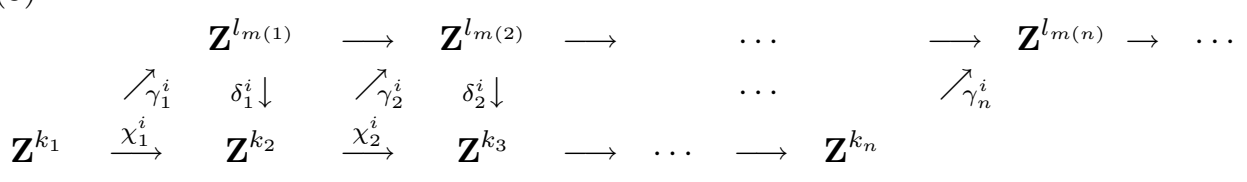

and

$$
\begin{array}{ccccccc}
\mathbf{Z}^{k_{1}} \oplus \mathbf{Z}^{k_{1}} & \stackrel{\psi_{1}}{\longrightarrow} & \mathbf{Z}^{k_{2}} \oplus \mathbf{Z}^{k_{2}} & \stackrel{\psi_{2}}{\longrightarrow} & \cdots & \rightarrow & \mathbf{Z}^{k_{n}} \oplus \mathbf{Z}^{k_{n}} \\
\downarrow & & \downarrow & & \cdots & & \downarrow \\
E & = & E & = & \cdots & = & E
\end{array}
$$

and

$$
\begin{aligned}
& 0 \rightarrow \mathbf{Z}^{k_{j}} \stackrel{\iota}{\longrightarrow} \mathbf{Z}^{k_{j}} \oplus \mathbf{Z}^{k_{j}} \stackrel{\rho}{\longrightarrow} \mathbf{Z}^{k_{j}} \quad \rightarrow 0 \\
& \theta_{j}^{1} \downarrow \quad \zeta_{j} \downarrow \quad \downarrow \theta_{j}^{0} \\
& 0 \rightarrow K_{0}(A) \longrightarrow E \longrightarrow K_{1}(A) \rightarrow 0
\end{aligned}
$$

for $j=1,2, \ldots, n$, where $\psi_{j}$ is of the form $\psi_{j}(a, b)=\left(\chi_{j}^{1}(a)+\psi_{j}^{1}(b), \chi_{j}^{0}(b)\right)$, and $\theta_{j}^{i}=\bar{\xi}_{m(j)}^{i} \circ \gamma_{j}^{i}$.

Choose $a_{i} \in E$ such that $q\left(a_{i}\right)=\bar{\xi}_{m(n)}^{0}\left(e_{i}\right)$. By the commutativity of the diagram

$$
\begin{array}{ccccc}
\mathbf{Z}^{k_{n}} \oplus \mathbf{Z}^{k_{n}} & \stackrel{\rho}{\longrightarrow} & \mathbf{Z}^{k_{n}} & \stackrel{\gamma_{n}^{0}}{\longrightarrow} & \mathbf{Z}^{l_{m(n)}} \\
\zeta_{n} \downarrow & & \theta_{n}^{0} \downarrow & & \downarrow \bar{\xi}_{m(n)}^{0} \\
E & \stackrel{q}{\longrightarrow} & K_{0}(A) & = & K_{0}(A)
\end{array}
$$

we have for $i=1, \ldots, k_{n}$

$$
\zeta_{n}\left(0, e_{i}\right)-\sum_{j} \gamma_{n}^{0}(j, i) a_{j} \in \iota\left(K_{1}(A)\right)
$$

Since $K_{1}(A)=\bigcup_{n} \operatorname{im} \bar{\xi}_{n}^{1}$, these elements are contained in $\iota\left(\operatorname{im} \bar{\xi}_{m(n+1)}^{1}\right)$ for some $m(n+1)>m(n)$. Let $k_{n+1}=l_{m(n)} l_{m(n+1)}$, and index the coordinates in $\mathbf{Z}^{k_{n+1}}$ by $(s, t), s=1, \ldots, l_{m(n+1)}, t=1, \ldots, l_{m(n)}$. Define $\delta_{n}^{i}: \mathbf{Z}^{l_{m(n)}} \rightarrow \mathbf{Z}^{k_{n+1}}$ by

$$
\delta_{n}^{1}\left((s, t), t^{\prime}\right)= \begin{cases}\xi_{m(n+1) m(n)}^{1}(s, t) & \text { if } t=t^{\prime} \\ 0 & \text { otherwise }\end{cases}
$$

and

$$
\delta_{n}^{0}\left((s, t), t^{\prime}\right)= \begin{cases}1 & \text { if } t=t^{\prime} \\ 0 & \text { otherwise. }\end{cases}
$$

Define $\gamma_{n+1}^{i}: \mathbf{Z}^{k_{n+1}} \rightarrow \mathbf{Z}^{l_{m(n+1)}}$ by

$$
\gamma_{n+1}^{1}\left(s^{\prime},(s, t)\right)= \begin{cases}1 & \text { if } s=s^{\prime} \\ 0 & \text { otherwise }\end{cases}
$$

and

$$
\gamma_{n+1}^{0}\left(s^{\prime},(s, t)\right)= \begin{cases}\xi_{m(n+1) m(n)}^{0}(s, t) & \text { if } s=s^{\prime} \\ 0 & \text { otherwise }\end{cases}
$$

These are defined so that $\gamma_{n+1}^{i} \circ \delta_{n}^{i}=\xi_{m(n+1) m(n)}$, the quotient $\mathbf{Z}^{k_{n+1}} / \mathrm{im} \delta_{n}^{0}$ has no torsion, and $\gamma_{n+1}^{1}$ is surjective. Diagram (3) may now be extended in the following 
way:

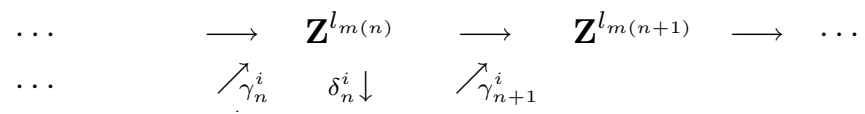

$$
\begin{aligned}
& \cdots \longrightarrow \mathbf{Z}^{k_{n}} \stackrel{\chi_{n}^{i}}{\longrightarrow} \mathbf{Z}^{k_{n+1}}
\end{aligned}
$$

where $\chi_{n}^{i}=\delta_{n}^{i} \gamma_{n}^{i}$.

Since $\delta_{n}^{0}\left(e_{t}\right)=\sum_{s} e_{(s, t)}$ and thus $\bar{\xi}_{m(n)}^{0}\left(e_{t}\right)=\theta_{n+1}^{0}\left(\sum_{s} e_{(s, t)}\right)$ (where $\theta_{n+1}^{i}=$ $\left.\bar{\xi}_{m(n+1)}^{i} \circ \gamma_{n+1}^{i}\right)$, there exist $a_{(s, t)} \in E$ such that $a_{t}=\sum_{s} a_{(s, t)}$ and $q\left(a_{(s, t)}\right)=$ $\theta_{n+1}^{0}\left(e_{(s, t)}\right)$. Since

$$
\bar{\xi}_{m(n+1)}^{1}\left(\mathbf{Z}^{l_{m(n+1)}}\right)=\theta_{n+1}^{1}\left(\mathbf{Z}^{k_{n+1}}\right)
$$

and

we obtain

$$
\chi_{n}^{0}((s, t), i)=\delta_{n}^{0}((s, t), t) \gamma_{n}^{0}(t, i)=\gamma_{n}^{0}(t, i),
$$

$$
\zeta_{n}\left(0, e_{i}\right)-\sum_{t} \gamma_{n}^{0}(t, i) \sum_{s} a_{(s, t)}=\zeta_{n}\left(0, e_{i}\right)-\sum_{s, t} \chi_{n}^{0}((s, t), i) a_{(s, t)} \in \theta_{n+1}^{1}\left(\mathbf{Z}^{k_{n+1}}\right) .
$$

Thus there exist $\left(\psi_{n}^{1}((s, t), i)\right)$ such that the left hand side equals

$$
\sum_{s, t} \psi_{n}^{1}((s, t), i) \theta_{n+1}^{1}\left(e_{(s, t)}\right) .
$$

Define $\psi_{n}: \mathbf{Z}^{k_{n}} \oplus \mathbf{Z}^{k_{n}} \rightarrow \mathbf{Z}^{k_{n+1}} \oplus \mathbf{Z}^{k_{n+1}}$ by

$$
\psi_{n}(a, b)=\left(\chi_{n}^{1}(a)+\psi_{n}^{1}(b), \chi_{n}^{0}(b)\right)
$$

and define $\zeta_{n+1}: \mathbf{Z}^{k_{n+1}} \oplus \mathbf{Z}^{k_{n+1}} \rightarrow E$ by

$$
\zeta_{n+1}\left(e_{(s, t)}, e_{\left(s^{\prime}, t^{\prime}\right)}\right)=\iota \circ \theta_{n+1}^{1}\left(e_{(s, t)}\right)+a_{\left(s^{\prime}, t^{\prime}\right)} .
$$

Then one checks that

$$
\begin{aligned}
& \mathbf{Z}^{k_{n}} \oplus \mathbf{Z}^{k_{n}} \quad \stackrel{\psi_{n}}{\longrightarrow} \quad \mathbf{Z}^{k_{n+1}} \oplus \mathbf{Z}^{k_{n+1}} \\
& \zeta_{n} \downarrow \quad \zeta_{n+1} \downarrow
\end{aligned}
$$

and

$$
\begin{aligned}
& 0 \quad \rightarrow \quad \mathbf{Z}^{k_{n+1}} \stackrel{\iota}{\longrightarrow} \mathbf{Z}^{k_{n+1}} \oplus \mathbf{Z}^{k_{n+1}} \stackrel{\rho}{\longrightarrow} \mathbf{Z}^{k_{n+1}} \quad \rightarrow 0 \\
& \theta_{n+1}^{1} \downarrow \quad \zeta_{n+1} \downarrow \quad \downarrow \theta_{n+1}^{0} \\
& 0 \rightarrow K_{1}(A) \longrightarrow E \quad \longrightarrow \quad K_{0}(A) \rightarrow 0
\end{aligned}
$$

are commutative.

For example, since

$$
\begin{aligned}
\psi_{n}\left(e_{i}, 0\right) & =\left(\sum_{s, t} \chi_{n}^{1}((s, t), i) e_{(s, t)}, 0\right), \\
\chi_{n}^{1}((s, t), i) & =\xi_{m(n+1) m(n)}^{1}(s, t) \gamma_{n}^{1}(t, i), \\
\theta_{n+1}^{1}\left(e_{(s, t)}\right) & =\bar{\xi}_{m(n+1)}^{1}\left(e_{s}\right),
\end{aligned}
$$

it follows that

$$
\begin{aligned}
\zeta_{n+1} \circ \psi_{n}\left(e_{i}, 0\right) & =\sum_{s, t} \chi_{n}^{1}((s, t), i) \iota \circ \theta_{n+1}^{1}\left(e_{(s, t)}\right)=\sum_{t} \gamma_{n}^{1}(t, i) \iota \circ \bar{\xi}_{m(n)}^{1}\left(e_{t}\right) \\
& =\iota \circ \bar{\xi}_{m(n)}^{1} \circ \gamma_{n}^{1}\left(e_{i}\right)=\iota \circ \theta_{n}^{1}\left(e_{i}\right)=\zeta_{n}\left(e_{i}, 0\right) .
\end{aligned}
$$


3.11. Proof of the theorem for the case $i=0$. By Lemma 3.10 we may assume that the extension

$$
0 \rightarrow K_{1}(A) \rightarrow E \rightarrow K_{0}(A) \rightarrow 0
$$

is obtained as the inductive limit of the sequence

$$
\begin{aligned}
& 0 \rightarrow \underset{\chi_{1}^{1} \downarrow}{\mathbf{Z}^{k_{1}}} \stackrel{\iota}{\longrightarrow} \underset{\chi_{1}^{1} \downarrow}{\mathbf{Z}^{k_{1}}} \underset{\swarrow_{\psi_{1}^{1}}}{\oplus} \mathbf{Z}^{k_{1}^{0}} \stackrel{\rho}{\longrightarrow} \quad \begin{array}{c}
\mathbf{Z}^{k_{1}} \\
\downarrow \chi_{1}^{0}
\end{array} \quad \rightarrow \quad 0 \\
& 0 \rightarrow \mathbf{Z}^{k_{2}} \stackrel{\iota}{\longrightarrow} \mathbf{Z}^{k_{2}} \oplus \mathbf{Z}^{k_{2}} \stackrel{\rho}{\longrightarrow} \mathbf{Z}^{k_{2}} \rightarrow 0 \\
& \begin{array}{ccccccc} 
& \downarrow & \downarrow & \swarrow & \downarrow & \downarrow \\
\vdots & \vdots & \vdots & \vdots & \vdots & \vdots & \vdots
\end{array}
\end{aligned}
$$

By passing to a subsequence we may assume that $M\left(\chi_{n+1}^{0}\right) \geq \max \left(2 L\left(\chi_{n}^{1}\right), 4\right)$; this will ensure that the inductive limit algebra to be constructed has real rank zero. As in 1.6, we construct an inductive system for $A$ with

$$
A_{n}=\bigoplus_{k=1}^{k_{n}} M_{[n, k]} \otimes C(\mathbf{T})
$$

and $\varphi_{n}: A_{n} \rightarrow A_{n+1}$ so that $\varphi_{n}$ induces $\chi_{n}^{i}: K_{i}\left(A_{n}\right) \rightarrow K_{i}\left(A_{n+1}\right)$ and the partial embeddings are standard embeddings of type $\left(\chi_{n}^{0}(i, j), \chi_{n}^{1}(i, j)\right)$.

We define $\alpha$ using a sequence of unitaries $u_{n} \in A_{n}$ chosen recursively such that

$$
\left[\varphi_{n}\left(p_{n, j}\right) u_{n+1} p_{n+1, i} \varphi_{n}\left(u_{n}^{*} p_{n, j}\right)\right]_{1}=[n, j] \psi_{n}^{1}(i, j),
$$

where $[\cdot]_{1}$ denotes the class of a unitary in

$$
K_{1}\left(\varphi_{n}\left(p_{n, j}\right) A_{n+1, i} \varphi_{n}\left(p_{n, j}\right)\right) \cong \mathbf{Z}
$$

(see 2.3). Set $q_{i j}^{n+1}=\varphi_{n}\left(p_{n, j}\right) p_{n+1, i}$ and put

$$
\begin{aligned}
& A_{i j}^{n+1}=q_{i j}^{n+1} A_{n+1} q_{i j}^{n+1}, \\
& B_{i j}^{n+1}=q_{i j}^{n+1} \varphi_{n}\left(A_{n}\right)^{\prime} \cap A_{n+1} q_{i j}^{n+1} ;
\end{aligned}
$$

one checks that, under the identification $K_{1}\left(A_{i j}^{n+1}\right) \cong \mathbf{Z}$, the range of the map $K_{1}\left(B_{i j}^{n+1}\right) \rightarrow K_{1}\left(A_{i j}^{n+1}\right)$ (induced by the embedding $\left.B_{i j}^{n+1} \subset A_{i j}^{n+1}\right)$ is $[n, j] \mathbf{Z}$. Set $u_{1}=1$; given $u_{n}$, construct $u_{n+1}$ as follows. Choose a unitary $w_{i j}^{n+1} \in B_{i j}^{n+1} \subset A_{i j}^{n+1}$ such that $\left[w_{i j}^{n+1}\right]_{1}=[n, j] \psi_{n}^{1}(i, j)$. Set $u_{n+1}=\left(\bigoplus w_{i j}^{n+1}\right) u_{n}$; since $\bigoplus w_{i j}^{n+1} \in$ $A_{n+1} \cap \varphi_{n}\left(A_{n}\right)^{\prime}$, one has $\alpha_{n+1} \circ \varphi_{n}=\varphi_{n} \circ \alpha_{n}$ (where $\left.\alpha_{n}=\operatorname{Ad} u_{n}\right)$. Then $\alpha=$ $\lim \operatorname{Ad} \bar{\varphi}_{n}\left(u_{n}\right)$ has the desired properties.

3.12. Corollary. Let $A$ be a simple unital AT algebra of real rank zero and $g \in$ $K K(A, A)$ such that $\gamma(g)=0$. Then there is an approximately inner automorphism $\alpha$ of $A$ such that $g=1-[\alpha]$.

Proof. This follows immediately from the preceding theorem and the universal coefficient theorem (see [RS, 1.17]).

3.13. Corollary. Let $A$ and $B$ be simple unital $A \mathbf{T}$ algebras of real rank zero and $g$ be an invertible element in $K K(A, B)$ such that $\gamma(g)$ preserves positivity and the class of the unit. Then there is an isomorphism $\varphi: A \rightarrow B$ such that $g=[\varphi]$. 
Proof. Since $g$ is invertible, $\gamma(g)_{i} \in \operatorname{Hom}\left(K_{i}(A), K_{i}(B)\right)$ is an isomorphism for $i=0,1$; further, $\gamma_{0}\left(\left[1_{A}\right]\right)=\left[1_{B}\right]$ and $\gamma_{0}\left(K_{0}(A)^{+}\right) \subset K_{0}(B)^{+}$. Hence, by Elliott's classification theorem (cf. [E, 7.1]) there is an isomorphism, $\psi: A \rightarrow B$, such that $\gamma(g)=\gamma([\psi])$. By the preceding corollary it follows that there is an approximately inner automorphism $\alpha$ such that $g=[\psi \circ \alpha]$; set $\varphi=\psi \circ \alpha$.

3.14. Remark. Let $\operatorname{Inn}(A)$ denote the group of inner automorphisms of a $\mathrm{C}^{*}$-algebra $A$ and let $\overline{\operatorname{Inn}}(A)$ denote its closure, the group of approximately inner automorphisms of $A$; further, let $\overline{\operatorname{Inn}}_{0}(A)$ denote the subgroup of approximately inner automorphisms which can be approximated by automorphisms of the form $\operatorname{Ad} u$ with $u$ in the connected component of the unitary group. In [ER, 4.5] Elliott and Rørdam show that for $A$ a simple unital $A \mathbf{T}$ algebra of real rank zero one has,

$$
\overline{\operatorname{Inn}}(A) / \overline{\operatorname{Inn}}_{0}(A) \cong \lim _{\leftarrow} K_{1}(A) / n_{j} K_{1}(A)
$$

where $n_{j}$ ranges over the directed set of divisors of [1] in $K_{0}(A)$ (we assume that $\left.n_{j} \mid n_{j+1}\right)$ and the isomorphism commutes with the canonical homomorphism from $K_{1}(A)$ to both groups. They ask whether $\overline{\operatorname{Inn}}_{0}(A)$ must be arc-connected. By construction the $\alpha$ we use in the proof of Theorem 3.1 for the case $i=1$ (see 3.8) is in $\overline{\operatorname{Inn}}_{0}(A)$. Thus, to answer the question in the negative it suffices to find a simple unital $A \mathbf{T}$ algebra of real rank zero for which $\operatorname{Ext}\left(K_{1}(A), K_{0}(A)\right) \neq 0$; there is then an $\alpha \in \overline{\operatorname{Inn}}_{0}(A)$ for which $\eta_{1}(\alpha) \neq 0$ (note that $\eta_{1}$ is a homotopy invariant). It is also possible that $\eta_{0}(\alpha) \neq 0$ for such $\alpha$, as shown below.

An outer form of the Elliott-Rørdam invariant for $\alpha \in \overline{\operatorname{Inn}}(A)$ may be obtained from $\eta_{0}(\alpha)$ as follows: taking the quotient of both sides of the above isomorphism (5) by the image of $K_{1}(A)$, one obtains

$$
\begin{aligned}
(\overline{\operatorname{Inn}}(A) / \operatorname{Inn}(A)) /\left(\overline{\operatorname{Inn}}_{0}(A) / \overline{\operatorname{Inn}}_{0}(A) \cap \operatorname{Inn}(A)\right) & \cong\left(\lim _{\leftarrow} K_{1}(A) / n_{j} K_{1}(A)\right) / K_{1}(A) \\
& \cong \lim _{\leftarrow}^{1} n_{j} K_{1}(A) .
\end{aligned}
$$

With $n_{j}$ as above, let $D$ denote the inductive $\operatorname{limit} \lim D_{j}$, where $D_{j}=\mathbf{Z}$ and the map $D_{j} \rightarrow D_{j+1}$ is given by multiplication by $n_{j+1} / n_{j}$; we identify $D$ with the divisible hull of $\mathbf{Z}\left[1_{A}\right]$ in $K_{0}(A)$ via the map $\iota: D \rightarrow K_{0}(A)$. It follows that

$$
\lim ^{1} n_{j} K_{1}(A) \cong \lim _{\leftarrow}^{1} \operatorname{Hom}\left(D_{j}, K_{1}(A)\right) \cong \operatorname{Ext}\left(D, K_{1}(A)\right) \text {. }
$$

With identifications as above the Elliott-Rørdam invariant for $\alpha$ is $\iota^{*}\left(\eta_{0}(\alpha)\right)$.

\section{REFERENCES}

[B] B. Blackadar, K-theory for operator algebras, MSRI Publications 5, Springer-Verlag, New York, 1986. MR 88g:46082

[BBEK] B. Blackadar, O. Bratteli, G. A. Elliott and A. Kumjian, Reduction of real rank in inductive limits of $C^{*}$-algebras, Math. Ann. 292 (1992) 111-126. MR 93a:46112

[D] V. Deaconu, A path model for circle algebras, J. Operator Theory 34 (1995) 57-89. MR 96m: 46113

[E] G. A. Elliott, On the classification of $C^{*}$-algebras of real rank zero, J. Reine Angew. Math. 443 (1993) 179-219. MR 94i:46074

[ER] G. A. Elliott and M. Rørdam, The automorphism group of the irrational rotation $C^{*}$ algebra, Commun. Math. Phys. 155 (1993) 3-26. MR 94j:46059

[Ex] R. Exel, The soft torus and applications to almost commuting matrices, Pacific J. Math. 160 (1993) 207-217. MR 94f:46091

[EL] R. Exel and T. Loring, Invariants of almost commuting matrices, J. Funct. Anal. 95 (1991) 364-376. MR 92a:46083 
[Ro] J. E. Roos, Sur les foncteurs dérivés de $\underbrace{}_{\leftarrow}$. Applications, C. R. Acad. Sci. Paris 252 (1961) 3702-3704. MR 24:A1938

[RS] J. Rosenberg and C. Schochet, The Künneth theorem and the universal coefficient theorem for Kasparov's generalized K-functor, Duke Math. J. 55 (1987) 431-474. MR 88i:46091

[Rø] M. Rørdam, Classification of certain infinite simple $C^{*}$-algebras, J. Funct. Anal. 131 (1995) 415-458. MR 96e:46080a

Department of Mathematics, Hokkaido University, Sapporo 060 Japan

E-mail address: kishi@math.sci.hokudai.ac.jp

Department of Mathematics, University of Nevada, Reno, Nevada 89557

E-mail address: alex@unv.edu 